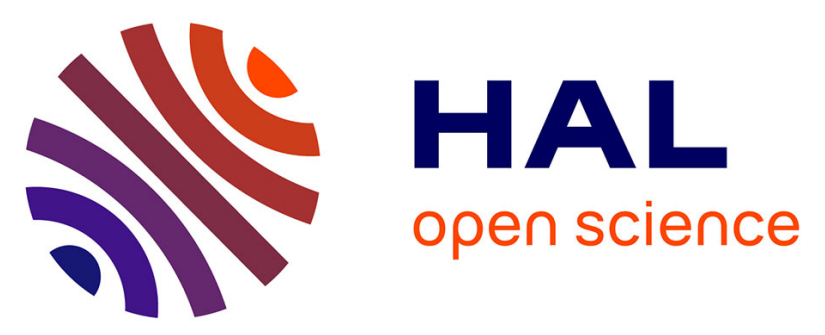

\title{
Robust FDI for fault-tolerant thrust allocation with application to spacecraft rendezvous
}

\author{
Robert Fonod, David Henry, Catherine Charbonnel, Eric Bornschlegl, \\ Damiana Losa, Samir Bennani
}

\section{- To cite this version:}

Robert Fonod, David Henry, Catherine Charbonnel, Eric Bornschlegl, Damiana Losa, et al.. Robust FDI for fault-tolerant thrust allocation with application to spacecraft rendezvous. Control Engineering Practice, 2015, 42, pp.12-27. 10.1016/j.conengprac.2015.05.004 . hal-01152116

\author{
HAL Id: hal-01152116 \\ https://hal.science/hal-01152116
}

Submitted on 29 Jan 2016

HAL is a multi-disciplinary open access archive for the deposit and dissemination of scientific research documents, whether they are published or not. The documents may come from teaching and research institutions in France or abroad, or from public or private research centers.
L'archive ouverte pluridisciplinaire HAL, est destinée au dépôt et à la diffusion de documents scientifiques de niveau recherche, publiés ou non, émanant des établissements d'enseignement et de recherche français ou étrangers, des laboratoires publics ou privés. 


\title{
Robust FDI for fault-tolerant thrust allocation with application to spacecraft rendezvous ${ }^{\natural}$
}

\author{
Robert Fonod $^{\mathrm{a}, 1, *}$, David Henry ${ }^{\mathrm{a}}$, Catherine Charbonnel $^{\mathrm{b}}$, Eric Bornschlegl ${ }^{\mathrm{c}}$, Damiana Losa ${ }^{\mathrm{b}}$, \\ Samir Bennanic \\ $\natural$ : This paper is an extended version with new methodological and applicative results of the work entitled \\ "Thruster Fault Detection, Isolation and Accommodation for an Autonomous Spacecraft" presented at the 19th \\ IFAC World Congress held in Cape Town, August 2014 \\ ${ }^{a}$ University of Bordeaux, IMS Lab. UMR CNRS n.5218, F-33400 Talence, France \\ ${ }^{b}$ Thales Alenia Space, F-06156 Cannes La Bocca, France \\ ${ }^{c}$ European Space Agency, ESTEC, 2200 AG Noordwik, The Netherlands
}

\begin{abstract}
This paper deals with the design and validation of an active fault-tolerant control system to detect, isolate and accommodate a single thruster fault affecting the thruster-based propulsion system of an autonomous spacecraft. The proposed method consists of a fault detector for robust and quick fault detection, a two-stage hierarchical isolation strategy for fault isolation, and an online control allocation unit scheduled by the isolation scheme for fault tolerance. A new factorization approach for the uncertain inertia matrix inverse is proposed. Thanks to this factorization, a novel robust Nonlinear Unknown Input Observers (NUIO) approach is proposed based on LMIs which ensure maximization of the admissible Lipschitz constant while at the same time satisfying an $\mathcal{L}_{2}$ gain bound and some constraints on the observer dynamics. At the first stage of the isolation scheme, a bank of NUIOs is used to identify a subset of possible faulty thrusters. Then, at the second stage, an EKF is introduced to estimate the torque bias directions. Using these directions, jointly with the detector's residual and the information obtained from the first stage, a set of explicit rules is derived to unambiguously isolate the faulty thruster. A Monte Carlo campaign, based on a simulator developed by Thales Alenia Space industries, is conducted in the context of a terminal rendezvous phase of the Mars Sample Return mission. Mission oriented criteria demonstrate that the proposed strategy is able to cope with a large class of realistic thruster faults and to achieve mission success.

*Corresponding author. Tel.: +33-540002419 - Fax.: +33-540006644

Email addresses: robert.fonod@ims-bordeaux.fr (Robert Fonod), david.henry@ims-bordeaux.fr (David Henry), catherine.charbonnel@thalesaleniaspace.com (Catherine Charbonnel), eric.bornschlegl@esa.int (Eric Bornschlegl), damiana.losa@thalesaleniaspace.com (Damiana Losa), samir.bennani@esa.int (Samir Bennani)

${ }^{1}$ Present address: Technion - Israel Institute of Technology, Department of Aerospace Engineering, Technion City, 32000 Haifa, Israel
\end{abstract}


Keywords: Fault detection and isolation, fault-tolerant control, unknown input observer, linear matrix inequalities, control allocation, space rendezvous mission, matrix factorization.

\section{Introduction}

\subsection{Context and Motivations}

The research work addressed in this paper draws expertise from actions undertaken between the European Space Agency (ESA), the Thales Alenia Space (TAS) industry and the IMS laboratory (laboratoire de l'Intégration du Matériau au Système) which develop new generations of integrated Guidance, Navigation and Control (GNC) algorithms for spacecraft with fault diagnosis and fault tolerance capabilities.

The reference space mission considered in this paper is the ESA Mars Sample Return (MSR) mission, see (Beaty et al., 2008) for details. This deep space mission consists of two vehicles directly injected towards Mars by launchers. The first module enters the Martian atmosphere (entry phase), lands on the Mars surface, fetches a Martian sample and then takes-off to reach a low Mars orbit. Meanwhile the second module inserts directly around Mars, then catches the sample (capture of the orbiting sample released by the first module), and finally comes back to Earth ejecting the sample into Earth atmosphere with the Earth Reentry Capsule (ERC). The work reported in this paper focuses on the terminal rendezvous phase which corresponds to the last few hundred meters until the capture on the Mars orbit. The chaser vehicle is the MSR orbiter, while the target is a diameter spherical container.

During the terminal rendezvous, the control of the attitude and the position of the chaser is continuous and applied by thrusters. The control unit uses different types of sensors, namely Inertial Measurement Units (IMU), Star Trackers (STR) and a Light Detection And Ranging (LIDAR) sensor. The set of sensors and actuators during the terminal rendezvous is minimized to reduce the risk of fault occurrence and to reduce the power consumption and mass. The attitude is controlled in order to keep the orbiting sample within the LIDAR field of view. The position is controlled in order to approach the orbiting sample along its velocity axis. Then, just before the capture, the guidance is modified in order to align the capture mechanism with the orbiting sample, i.e. the target.

Following recent studies (Tafazoli, 2009; HARVD - Final Presentation), thruster faults account for approximatively one quarter of all Attitude and Orbit Control System (AOCS) failures. It seems obvious that they can have a serious impact on the spacecraft's ability to fulfil its mission. For instance, a hardover type failure (thruster stucks open), it could lead to a drastic increase of the propellant consumption which is already very constrained by the travel to Mars. Dramatic consequences can occur, e.g. already in-placed GNC may not compensate such faults, possibly leading the chaser to lose the attitude and/or the position of the sample container.

The work addressed in this paper is concerned by the development of a model-based Fault Detection and Isolation (FDI) scheme for a Fault-tolerant Control (FTC) of the thruster which 
equip the MSR chaser propulsion system. The investigated faults have been defined in accordance with the industrial partners and follow both the ESA and TAS requirements and their experiences. Four cases are investigated: $i$ ) thruster opening at 100\% (providing maximum force regardless of the demand and being very propellant consuming) $i i$ ) thruster closing itself (faulty thruster does not generate any thrust regardless of the demanded command by the control authority) iii) bi-propellant leakage and iv) loss of efficiency (thrust loss).

\subsection{Related Work and Limitations}

In terms of model-based FDI, numerous techniques have been studied in the past decades in the academic community, see (Patton et al., 2000; Blanke et al., 2006; Ding, 2013) and references therein for good surveys. The still growing interest of potential applications in aerospace systems has been demonstrated by recent publications. With regards to the problem of spacecraft thruster fault diagnosis, one can mention the work of Chen and Saif (2007) that proposed an iterative learning observer to achieve estimation of time-varying thruster faults. Wu and Saif (2009) proposed the same approach jointly with a sliding mode technique. The work reported in (Patton et al., 2006, 2008, 2010) addressed the Mars Express mission. The proposed approach is based on both state estimation of an accurate linear model for the satellite system and unknown input decoupling to achieve robust FDI in the presence of dynamic uncertainty during main engine deployment. The work reported in (Henry et al., 2011; Fonod et al., 2014a; LePeuvédic et al., 2014; Fonod et al., 2015) addressed the problem of thruster fault diagnosis of the MSR orbiter during the terminal rendezvous phase. Henry et al. (2011) proposed a method based on a $H(0)$ filter with robust poles assignment technique. Fonod et al. (2015) approached the same problem using an Eigenstructure Assignment (EA) technique, whereas LePeuvédic et al. (2014) proposed a robust $H_{\infty} / H_{-}$filter in combination with a bank of thruster-direction decoupling observers. Similarly in (Falcoz et al., 2010a,b), the $H_{\infty} / H_{-}$approach was exploited for the micro-Newton colloidal thrusters during the experiment phase of the LISA Pathfinder mission. $H_{\infty} / H_{-}$filter-based strategies have been proposed in (Grenaille et al., 2004; Henry, 2008a) to diagnose the Field Emission Electric Propulsion (FEEP) thrusters of the Microscope satellite.

In the case of an overactuated spacecraft, the cornerstone of the FDI unit is the isolation logic. It must be accurate and robust enough to uncover the faulty thruster among thrusters which are very closely co-aligned and it also must be able to cover a large class of realistic faults. Posch et al. (2013) proposed a torque bias vector matching isolation method. In this approach, the torque bias is estimated using an Extend Kalman Filter (EKF) and directly matched with the torque directions of each thruster. The main drawback of this approach is that it is unable to consider a thruster configuration where some thrusters generate the same or very similar torques. Similar idea has been presented in (Alwi et al., 2010), where instead of estimating the torque bias, the sliding mode injection term is matched with the thruster directions. This method has similar drawbacks as the previous method, additionally, the isolation performance strongly depends on the measurement noise. In (Henry et al., 2011; Fonod et al., 2015), a cross-correlation test between the residual and the associated thruster opening rates was considered. This approach however lacks the ability to consider both "open-type" 
and "closed-type" thrusters faults at the same time (for fault classification, see Section 2.1). Moreover, in the aerospace systems, the true inertia matrix is newer known precisely on-board. Therefore, controllers are always validated in presence of uncertainty on the inertia to confront modelling errors. Similarly, in terms of FDI, it is of paramount interest to analyse, and most importantly, to incorporate the effects of the uncertain inertia within the FDI design.

In terms of FTC methods, the interested reader shall refer to (Blanke et al., 2006; Zhang and Jiang, 2008; Noura et al., 2009). These techniques can be in general classified into two main categories: passive FTC and active FTC. Passive FTC relies on robust control concepts, whereas active FTC methods act on the system component failures actively by re-designing the controller so that the stability and acceptable performance of the entire system is maintained. The most famous active FTC strategies are the pseudoinverse methods (Ostroff, 1985; Caglayan et al., 1988; Gao and Antsaklis, 1991; Bajpai et al., 2001), recently revisited by Staroswiecki (2005), the Linear Quadratic (LQ) approach (Looze et al., 1985; Josh, 1987; Veillette, 1995; Staroswiecki et al., 2007), the EA technique (Jiang, 1994; Zhao and Jiang, 1998; Zhang and Jiang, 2001), the adaptive control approach (Bodson and Groszkiewicz, 1997; Tao et al., 2002; Zhang et al., 2004), the Model Predictive Control (MPC) approach (Camacho and Bordons, 1999; Maciejowski, 2002; Hartley et al., 2012), and most recently the supervisory approach (Yang et al., 2012; Efimov et al., 2013).

The problem of designing an active FTC system for thruster faults has been rarely studied for space systems (or very few papers have been published). The already in-placed industrycertified controllers are designed to be robust and to achieve a predetermined performance level in a fault safe situation. The Control Allocation (CA) technique is probably the most "ready to be implemented" FTC approach for aerospace systems. The major reason is that the computational burden is very close or within the limits of today's off-the-shelf embedded computer systems. Moreover, in some cases the CA approach does not require any change in the nominal controller which is a great advantage from an industrial point of view. Several application of the CA from the aerospace community can be found in (Bodson, 2002; Page and Steinberg, 2002; Jin et al., 2006; Henry, 2008b; Oppenheimer et al., 2010; Boada et al., 2010; $\mathrm{Fu}$ et al., 2011). For instance, a SIMPLEX-based method has been reacently implemented in the Automated Transfer Vehicle (ATV) developed by EADS Astrium Space Transportation, to carry out a prescribed set of thruster faults.

Most CA algorithms assume a linear effector model in the form of a matrix, i.e. the thruster configuration matrix whose elements (columns) are the influence coefficients defining how each thruster affects each component of the force and moment vector applied to the spacecraft. Thus, CA is fundamentally concerned by the inverse computation of the thruster configuration matrix. Since this matrix has more columns than rows, there exists an infinite number of solutions. However, by minimizing some "measure" of it, it is possible to have a unique solution. Actuator faults can then be tackled by a CA principle so that it is not required to re-design the nominal controller itself. A consequence is that CA can be used as a FTC solution with a little extra effort on the existing CA techniques. Alwi and Edwards (2008) exploits this idea using sliding mode techniques. 


\subsection{Proposed Approach and Contributions}

This paper addresses the design and validation of a complete FDI/FTC system for the aforementioned thruster fault scenarios. The proposed method consists of: i) a fault detector for robust and quick fault detection, ii) a two-stage hierarchical isolation strategy for faulty thruster isolation and iii) an online CA unit scheduled by the isolation scheme for fault tolerance. The utilized fault detector design follows the developments introduced in (Fonod et al., 2013). This detector offers enhanced robustness against time-varying input delays. The original idea of the two-stage isolation strategy proposed in this paper initiates from (Fonod et al., 2014a), where a bank of asymptotically stable Nonlinear Unknown Input Observers (NUIOs) has been used for the first stage and a simple residual vector matching approach for the second stage. Here, a bank of 5 robust NUIOs together with an EKF-based torque bias direction estimator is considered. A new factorization approach for the uncertain inertia matrix inverse is proposed. Thanks to this factorization, a novel robust NUIO design is proposed with bounded $\mathcal{L}_{2}$ gain from the system input to the estimation error. By this, the effect of the uncertain inertia on the state estimation error is attenuated. Additionally, it is shown that under some Lipschitz condition, it is possible to constrain the NUIO dynamics into a prescribed dynamic region using the notion of Linear Matrix Inequality (LMI) regions. The NUIO gains are obtained from the feasible solution to the LMI optimization problem, offering numerically tractable procedure to account jointly the observer dynamics constraint, the $\mathcal{L}_{2}$ specification, and the maximization of the admissible Lipschitz constant. As the outcome of the first stage, a subset of thrusters is identified as "possible faulty". For the second stage, an EKF is introduced to estimate the torque bias directions due to the thruster fault. Using these directions, the fault detector's residual and the information obtained from the first stage, a set of explicit rules is derived to unambiguously isolate the faulty thruster. These rules consist in evaluating the torque bias direction estimate with respect to the thruster torque directions and the detector's residual with respect to the thruster force directions of the already identified (faulty) thruster set, respectively. In specific cases, a sequential decision test is also used. As soon as the faulty thruster is identified, a control re-allocation algorithm is used to redistribute the control effort among the available healthy actuators, while at the same time disengaging the faulty one. Here, based on the precursor work of (Jin et al., 1995), a modified version of the Nonlinear Iterative Pseudoinverse Controller (NIPC) algorithm is presented. A complete Monte Carlo campaign is conducted in the context of the terminal rendezvous phase. Mission oriented criteria are evaluated to demonstrate the effectiveness of the proposed method subject to various sources of uncertainties, spatial disturbances, delays and imperfect navigation.

The paper is organized as follows. Section 2 is devoted to the thruster-based propulsion system of the chaser. It also introduces the considered actuator fault model. Sections 3 and 4 are dedicated to the FDI unit design. Section 5 deals with the FTC algorithm. Finally, a simulation campaign is conducted in Section 6 in the context of the terminal rendezvous phase. Concluding remarks are given in Section 7.

Notations: Let denote $\mathbb{R}, \mathbb{C}, \mathbb{Z}^{+}$, and $\mathbb{H}$ the set of real numbers, complex numbers, nonnegative integers, and the set of quaternions, respectively. The notation $\mathbb{R}^{m \times n}$ is used for real 
matrices of dimension $m \times n$. $\operatorname{diag}(\ldots)$ represents a block diagonal matrix. $\boldsymbol{I}$ and $\mathbf{0}$ represents the identity and zero matrix with the appropriate dimension, respectively. The symbol $\otimes, \times$, and $\cdot$ stands for the Kronecker, cross and dot product, respectively. The notation $\boldsymbol{P}>0(\boldsymbol{P}<0)$ means that $\boldsymbol{P}$ is a real symmetric and positive (negative) definite matrix. The notation $\Lambda(\boldsymbol{A})$ stands for the set of all eigenvalues and $\lambda_{\max }$ stands for the maximum eigenvalue of a square matrix $\boldsymbol{A}$, respectively. In symmetric block matrices, the symbol $*$ denotes an element that is induced by symmetry. $\|\cdot\|_{p}$ refers to either the p-norm of a vector or the induced matrix p-norm. If $p=2,\|\cdot\|_{p}$ is written without the subscript, i.e. $\|\cdot\|$. With $\mathcal{L}_{2}$ a space of all $\mathcal{L}$ ebensque measurable functions having a finite $\mathcal{L}_{2}$ norm $\|\boldsymbol{u}\|_{\ell 2}$ is denoted, where $\|\boldsymbol{u}\|_{\ell 2}^{2}=\int_{0}^{\infty}\|\boldsymbol{u}(t)\|^{2} d t$. $\mathcal{N}(\mu, \sigma)$ stays for the normal distribution with mean value $\mu$ and standard deviation $\sigma . \mathcal{U}(a, b)$ denotes the uniform distribution with boundaries $a$ and $b$.

\section{Background on Thruster-based Propulsion System and Fault Considerations}

The MSR chaser spacecraft is equipped with a chemical propulsion system composed of 12 thrusters. The thrusters are physically organised in four groups (see Fig. 1 for illustration) and are in charge of producing force a $\boldsymbol{F} \in \mathbb{R}^{3}$ and a torque $\boldsymbol{T} \in \mathbb{R}^{3}$ vector.
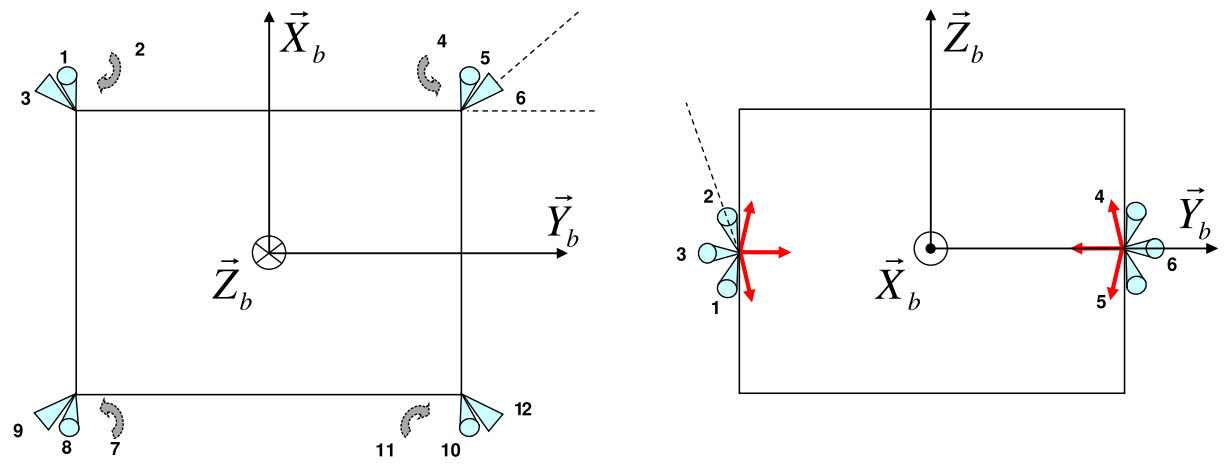

Figure 1: Thruster configuration of the chaser spacecraft ${ }^{2}$

Let denote $\mathcal{S}_{\text {all }}=\{1,2, \ldots 12\}$ the set of all the thruster indices. All thrusters have fixed directions $\boldsymbol{d}_{k} \in \mathbb{R}^{3}, \forall k \in \mathcal{S}_{\text {all }}$ and each one is able to produce a maximum thrust of $\left\|F_{T}\right\|=22 \mathrm{~N}$. The Chemical Propulsion Drive Electronics (CPDE) driving the thrusters, is initiating the opening of each thruster valve for the commanded duration $0 \leq u_{k} \leq 1, \forall k \in \mathcal{S}_{\text {all }}$ which are in fact scaled ON-times. The scaling is done versus the sampling period $T_{s}$ of the control unit and is defined according to $u_{i}\left(t_{k}\right)=T_{o n_{i}}\left(t_{k}\right) / T_{s}$, where $T_{o n_{i}}\left(t_{k}\right)$ is the actual/real firing duration (ON time) of the $i^{\text {th }}$ thruster at control cycle $t_{k}=k T_{s}$.

\footnotetext{
${ }^{2}$ The considered thruster configuration in this paper is a special one designed by TAS to study active FTC strategies.
} 
The propulsion system is obviously a source of uncertainty in the system. The transfer function

$$
\mathcal{H}(s)=e^{-\tau(t) s}
$$

aims to model the effect of the unknown time-varying delays induced by the CPDE and the uncertainties on the thruster rise times (see Pettazzi et al. (2009)). The delay $\tau(t)$ is assumed to be unknown and time-varying, but upper bounded by a known constant $\bar{\tau}$, i.e. $\tau(t) \leq \bar{\tau}$.

Let be $u_{k}(t-\tau(t))$ the commanded open duration of the $k^{t h}$ thruster delayed by $\tau(t)$. The net forces and torques generated by thrusters (in fault-free case) are given in the chaser body fixed frame $\mathcal{F}_{b}=\left\{O_{b}, \overrightarrow{\boldsymbol{X}}_{b}, \overrightarrow{\boldsymbol{Y}}_{b}, \overrightarrow{\boldsymbol{Z}}_{b}\right\}$ (see Fig. 1 for an illustration) according to

$$
\boldsymbol{F}(t)=\boldsymbol{B}_{F} \boldsymbol{u}(t-\tau(t)), \quad \boldsymbol{T}(t)=\boldsymbol{B}_{T} \boldsymbol{u}(t-\tau(t))
$$

In the above equation $\boldsymbol{u}(t)=\left[u_{1}(t), u_{2}(t), \ldots, u_{12}(t)\right]^{T}$, and

$$
\boldsymbol{B}_{F}=\left[\boldsymbol{b}_{F_{1}}, \boldsymbol{b}_{F_{2}}, \ldots, \boldsymbol{b}_{F_{12}}\right], \quad \boldsymbol{B}_{T}=\left[\boldsymbol{b}_{T_{1}}, \boldsymbol{b}_{T_{2}}, \ldots, \boldsymbol{b}_{T_{12}}\right]
$$

are the thruster sensitivity (configuration) matrices with ${ }^{3}$

$$
\boldsymbol{b}_{F k}=-\boldsymbol{d}_{k}\left\|F_{T}\right\|, \quad \boldsymbol{b}_{T k}=\left(\boldsymbol{d}_{p k}-\boldsymbol{d}_{C o M}\right) \times \boldsymbol{b}_{F k}, \quad \forall k \in \mathcal{S}_{\text {all }}
$$

where $\boldsymbol{d}_{C o M} \in \mathbb{R}^{3}$ is the position vector of the Center of Mass (CoM) from the center of the chaser geometrical frame $\mathcal{F}_{g}$, and $\boldsymbol{d}_{p k} \in \mathbb{R}^{3}, \forall k \in \mathcal{S}_{\text {all }}$ are the position (location) vectors of the thrusters, all given in $\mathcal{F}_{g}$.

By analysing the matrices $\boldsymbol{B}_{F}$ and $\boldsymbol{B}_{T}$ in terms of directional properties, the following can be concluded: the torque directions of the thrusters having index inside the sets $\mathcal{S}_{T k}, k=1, \ldots, 4$ are the same and those having index inside the set $\mathcal{S}_{T 5}$ are similar. In our case, the above subsets are defined as follows:

$$
\begin{array}{ll}
\mathcal{S}_{T 1}=\{1,11\}, & \mathcal{S}_{T 3}=\{4,8\}, \quad \mathcal{S}_{T 5}=\{3,6,9,12\} \\
\mathcal{S}_{T 2}=\{2,10\}, & \mathcal{S}_{T 4}=\{5,7\},
\end{array}
$$

In terms of force directions, the following is revealed

$$
\begin{array}{lll}
\boldsymbol{b}_{F 1}=-\boldsymbol{b}_{F 11}, & \boldsymbol{b}_{F 4}=-\boldsymbol{b}_{F 8}, & \boldsymbol{b}_{F 3}=-\boldsymbol{b}_{F 12} \\
\boldsymbol{b}_{F 2}=-\boldsymbol{b}_{F 10}, & \boldsymbol{b}_{F 5}=-\boldsymbol{b}_{F 7}, & \boldsymbol{b}_{F 6}=-\boldsymbol{b}_{F 9}
\end{array}
$$

which means that the thruster pairs of the sets $\mathcal{S}_{T k}, k=1, \ldots, 4$ produce exactly opposite forces. The last thruster group, i.e. $\mathcal{S}_{T 5}$, has the following properties

$$
\boldsymbol{b}_{F 3} \cdot \boldsymbol{b}_{F 6}=0, \quad \boldsymbol{b}_{T 3} \approx-\boldsymbol{b}_{T 6} \approx-\boldsymbol{b}_{T 9} \approx \boldsymbol{b}_{T 12}
$$

Relations in (6) mean that thrusters belonging to $\mathcal{S}_{T 5}$ group produce a) forces perpendicular to the forces of their neighbours b) nearly collinear torques. The directional properties given by (4)-(6) will be later used to derive an explicit fault isolation strategy.

\footnotetext{
${ }^{3}$ Numerical values with regards to the spacecraft geometry are omitted for confidentiality reasons.
} 


\subsection{Thruster Fault Modelling}

With regards to the possible faults occurring in the thruster-based propulsion system, the focus is on the so-called "open-type" (fully open or leaking thruster) and "closed-type" (blockedclosed thruster or loss of efficiency) faults. These faults have been defined in accordance with the industrial partners and follow both the TAS and ESA experiences. The following mathematical model can be used to describe these faults

$$
\varphi_{k}(t)= \begin{cases}\max \left\{u_{k}(t), m_{\text {leak }}\right\} & \text { if open-type } \\ \left(1-m_{\text {loss }}\right) u_{k}(t) & \text { if closed-type }\end{cases}
$$

where the index " $k$ " refers to the $k^{\text {th }}$ thruster. In this formalism, $0<m_{\text {leak }}<1$ models a leakage fault and $0<m_{\text {loss }}<1$ an efficiency loss fault. It is obvious that $m_{\text {leak }}=1$ refers to a fully open and $m_{\text {loss }}=1$ to a blocked-closed thruster fault, respectively.

Assuming no simultaneous faults, the considered thruster faults can be modelled in a multiplicative way according to (the index $f$ outlines the faulty case)

$$
\boldsymbol{u}_{f}(t)=(\boldsymbol{I}-\boldsymbol{\Psi}(t)) \boldsymbol{u}(t)
$$

with $\boldsymbol{\Psi}(t)=\operatorname{diag}\left(\psi_{1}(t), \ldots, \psi_{12}(t)\right)$, where $0 \leq \psi_{k}(t) \leq 1, \forall k \in \mathcal{S}_{\text {all }}$ are unknown. The status of the $k^{\text {th }}$ thruster is modelled by $\psi_{k}$ as follows

$$
\psi_{k}(t)= \begin{cases}0 & \text { if healthy } \\ 1-\varphi_{k}(t) / u_{k}(t) & \text { if faulty }\end{cases}
$$

where $\varphi_{k}$ allows to consider different fault scenarios.

\section{Design of the Robust Fault Detector}

The proposed fault detector consists of an observer-based residual generator and a sequential decision which evaluates the residual. The observer is designed based on the EA technique and uses a model of the relative position between the chaser and the target given in the local (target) frame. In (Fonod et al., 2015), it was shown that, in terms of robustness/sensitivity, the position model-based FDI scheme tends to achieve very similar FDI performances as a scheme based on a pure attitude model.

\subsection{Relative Position Model}

Consider the illustration of the rendezvous between the chaser and target spacecraft around Mars given by Fig. 2 where $\mathcal{F}_{l}=\left\{O_{T}, \overrightarrow{\boldsymbol{X}}_{l}, \overrightarrow{\boldsymbol{Y}}_{l}, \overrightarrow{\boldsymbol{Z}}_{l}\right\}$ is the local (target centred) reference frame oriented as shown in Fig. 2. During the rendezvous phase on a circular orbit, it is assumed that the chaser motion is due to the four following forces, all given in $\mathcal{F}_{l}$

- the Mars attraction force $\overrightarrow{\boldsymbol{F}}_{\boldsymbol{a}}=-m \frac{\mu}{\left((a+\xi)^{2}+\eta^{2}+\zeta^{2}\right)^{3 / 2}}\left((a+\xi) \overrightarrow{\boldsymbol{X}}_{l}+\eta \overrightarrow{\boldsymbol{Y}}_{l}+\zeta \overrightarrow{\boldsymbol{Z}}_{l}\right)$, where $\xi, \eta, \zeta$ denote the three components of the relative position vector $\Delta \boldsymbol{r}=[\xi, \eta, \zeta]^{T}$ of the chaser from the origin $O_{T}$ of the target frame $\mathcal{F}_{l}$, 


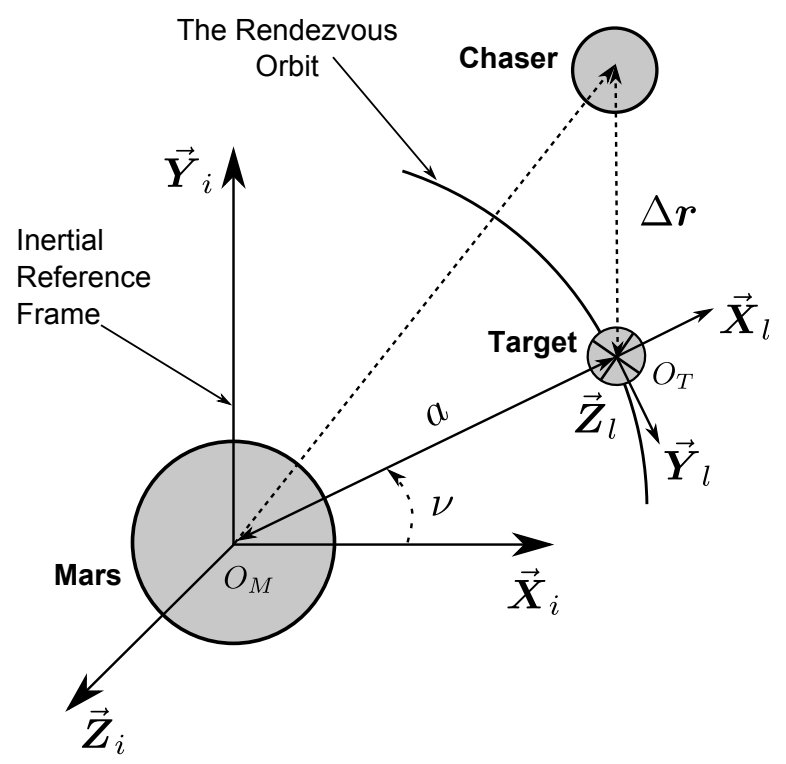

Figure 2: The Mars rendezvous orbit with the associated frames

- the centripetal force $\overrightarrow{\boldsymbol{F}}_{\boldsymbol{e}}=m\left(n^{2}(a+\xi) \overrightarrow{\boldsymbol{X}}_{l}+n^{2} \eta \overrightarrow{\boldsymbol{Y}}_{l}\right)$,

- the Coriolis force $\overrightarrow{\boldsymbol{F}}_{\boldsymbol{c}}=m\left(2 n \dot{\eta} \overrightarrow{\boldsymbol{X}}_{l}-2 n \dot{\xi} \overrightarrow{\boldsymbol{Y}}_{l}\right)$,

- the force due to the thruster-based propulsion system $\overrightarrow{\boldsymbol{F}}_{t}=F_{\xi} \overrightarrow{\boldsymbol{X}}_{l}+F_{\eta} \overrightarrow{\boldsymbol{Y}}_{l}+F_{\zeta} \overrightarrow{\boldsymbol{Z}}_{l}$. (This force vector is the one given by the equation $(2)$ expressed in $\mathcal{F}_{l}$.)

In these relations, $\mu=\mathcal{G} \cdot m_{M}{ }^{4}$ and $n=\dot{\nu}=\sqrt{\mu / a^{3}}$, where $a, m, \mathcal{G}$ and $m_{M}$ are the radius of the circular orbit of the target, the mass of the chaser, the universal gravitational constant and the mass of Mars, respectively. It can be verified that the above equations lead to a $6^{\text {th }}$ order nonlinear state space model whose state and force input vectors are given by $\boldsymbol{x}_{p}=[\xi \eta \zeta \dot{\xi} \dot{\eta} \dot{\zeta}]^{T}$ and $\boldsymbol{F}_{t}=\left[F_{\xi} F_{\eta} F_{\zeta}\right]^{T}$, respectively. Noting that the distance between the target and the chaser during the rendezvous phase is negligible compared to the radius of the target orbit, i.e. $\|\Delta \boldsymbol{r}\| \ll a$. It is then possible to derive the so called Hill-Clohessy-Wiltshire equations by means of a first order approximation of the nonlinear state space model (Sidi, 1997). Finally, introducing the fault model and the CPDE unknown time-varying delay $\tau(t)$ introduced in Section 2 , leads to the following linear $6^{\text {th }}$ order state space model of the chaser relative motion expressed in $\mathcal{F}_{l}$, both in fault-free $(\boldsymbol{\Psi}=\mathbf{0})$ and faulty $(\boldsymbol{\Psi} \neq \mathbf{0})$ situations, i.e.

$$
\begin{aligned}
& \dot{\boldsymbol{x}}_{p}(t)=\boldsymbol{A}_{p} \boldsymbol{x}_{p}(t)+\boldsymbol{B}_{p} \boldsymbol{R}\left(\hat{\boldsymbol{q}}_{t}(t), \hat{\boldsymbol{q}}_{c}(t)\right) \boldsymbol{B}_{F} \boldsymbol{u}_{f}(t-\tau(t)) \\
& \boldsymbol{y}_{p}(t)=\boldsymbol{C}_{p} \boldsymbol{x}_{p}(t)
\end{aligned}
$$

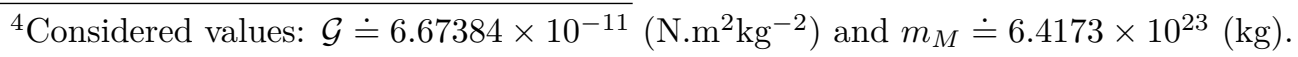




$$
\boldsymbol{A}_{p}=\left[\begin{array}{cccccc}
0 & 0 & 0 & 1 & 0 & 0 \\
0 & 0 & 0 & 0 & 1 & 0 \\
0 & 0 & 0 & 0 & 0 & 1 \\
3 n^{2} & 0 & 0 & 0 & 2 n & 0 \\
0 & 0 & 0 & -2 n & 0 & 0 \\
0 & 0 & -n^{2} & 0 & 0 & 0
\end{array}\right], \quad \boldsymbol{B}_{p}=\frac{1}{m}\left[\begin{array}{ccc}
0 & 0 & 0 \\
0 & 0 & 0 \\
0 & 0 & 0 \\
1 & 0 & 0 \\
0 & 1 & 0 \\
0 & 0 & 1
\end{array}\right], \quad \boldsymbol{C}_{p}=\left[\begin{array}{cccccc}
1 & 0 & 0 & 0 & 0 & 0 \\
0 & 1 & 0 & 0 & 0 & 0 \\
0 & 0 & 1 & 0 & 0 & 0
\end{array}\right]
$$

In (8), the rotation matrix $\boldsymbol{R}\left(\hat{\boldsymbol{q}}_{t}, \hat{\boldsymbol{q}}_{c}\right)$ is calculated from the attitude quaternion estimates of the chaser $\hat{\boldsymbol{q}}_{c} \in \mathbb{H}$ and target $\hat{\boldsymbol{q}}_{t} \in \mathbb{H}$. They rotate the force due to thrusters, i.e. $\boldsymbol{F}_{f}=$ $\boldsymbol{B}_{F} \boldsymbol{u}_{f}(t-\tau(t))$, from $\mathcal{F}_{b}$ into $\mathcal{F}_{l}$. These estimates are assumed to be available on-board since they are computed online by the navigation unit. The output vector $\boldsymbol{y}_{p}=\Delta \boldsymbol{r}=[\xi \eta \zeta]^{T}$ is the relative position expressed in $\mathcal{F}_{l}$. In the context of our study, this relative position is measured by the LIDAR device. Moreover, it is assumed that the navigation unit is decoupled from thruster faults, but providing noisy state estimates.

\subsection{Residual Generation and Evaluation}

The proposed residual generator is based on a full-order observer using the position model (8) and (9), introduced in the previous section. The observer is designed using the well known EA technique so that the residual vector output, i.e. the output estimation error weighted by a matrix $\boldsymbol{Q}$

$$
\boldsymbol{r}(t)=\boldsymbol{Q}\left(\boldsymbol{y}_{p}(t)-\boldsymbol{C}_{p} \hat{\boldsymbol{x}}_{p}(t)\right), \quad \boldsymbol{r}=\left[r_{1}, r_{2}, r_{3}\right]^{T}
$$

is (approximately) decoupled from the unwanted effects of the time-varying delay $\tau(t)$. Fonod et al. (2013) address this problem using two different approaches, i.e. using a Padé approximation and a Cayley-Hamilton theorem-based transformation. The earlier method is employed in this paper. The idea is to use the model (8)-(9) to generate the state estimate $\hat{\boldsymbol{x}}_{p}$ used to produce the residual vector $\boldsymbol{r}$. Since the EA technique is well mastered in the FDI community, technical developments are not considered in this paper. The interested reader can refer to e.g., (Patton et al., 2000; Blanke et al., 2006; Ding, 2013).

The proposed decision making rule is a slightly modified version of the scalar valued Generalized Likelihood Ratio (GLR) test for the variance (see e.g. Ding (2013)). The considered decision test $\varrho^{J_{t h}}$ is defined by

$$
\varrho^{J_{t h}}(t)= \begin{cases}1 & \text { if } S_{w}\left(\boldsymbol{r}\left(t_{k}\right)\right)>J_{t h} \Rightarrow \text { fault declared } \\ 0 & \text { if } S_{w}\left(\boldsymbol{r}\left(t_{k}\right)\right) \leq J_{t h} \Rightarrow \text { fault not present }\end{cases}
$$

with $S_{w}\left(\boldsymbol{r}\left(t_{k}\right)\right)=\sum_{i=1}^{3} w_{i} S_{i}\left(r_{i}\left(t_{k}\right)\right)$, where $w_{i} \geq 0, i=1,2,3$ being the normalized weight factors used to prioritize certain elements (axes) of the residual and $S_{i}\left(r_{i}\left(t_{k}\right)\right)$ is the estimated log likelihood of the GLR algorithm applied to the $i^{\text {th }}$ residual $r_{i}\left(t_{k}\right)$ evaluated at time instant $t=t_{k}=k T_{s}, k \in \mathbb{Z}^{+}$. In (11), the fixed threshold $J_{t h}$ is an additional design parameter, see (Basseville and Nikiforov, 1993) for discussion about its tuning. The fault is declared at time $t_{d}$, i.e.

$$
t_{d}=\underset{t \geq t_{0}}{\arg \inf }\left\{\varrho^{J_{t h}}(t)=1\right\}
$$


where $t_{0} \geq 0$ is time required for $\boldsymbol{r}$ to the achieve steady state (settle down) when $\Psi(t)=$ $\mathbf{0}, \forall t \in\left[0, t_{0}\right)$.

\section{Hierarchical Isolation Strategy}

Recalling the thruster configuration properties given by (4)-(6) and taking into account that thrusters cause both linear and rotational motions, a set of explicit rules can be derived to unambiguously isolate a single thruster fault. These rules are implemented on a hierarchical two-stage basis as follows:

i) The first stage utilizes a bank of five NUIOs based on the nonlinear model of the attitude dynamics. This bank is in charge of confining the faulty thruster into a single group $\mathcal{S}_{T j}, j=1, \ldots, 5$ (subset of thrusters), in other words, the task is to to find the faulty group index " $j$ ". An enhanced NUIO approach is adopted for this purposes because of its decoupling properties, adjustable error dynamics and ability to take into account both nonlinearities and uncertainties of the attitude dynamics,

ii) The second stage aims at uniquely isolating the faulty thruster index " $i$ " within the already identified subset, i.e. find $i \in \mathcal{S}_{T j}$. This stage uses jointly an EKF (being in charge of estimating the torque bias directions due to the fault), a torque bias matching approach and/or a Wald's sequential test, and finally a residual/force direction marching approach.

It is obvious that in case of (small) truster faults, the spacecraft attitude dynamics is more likely prone to dynamic deviations than the translation one. This gives the motivation to derive the first isolation rule using the angular velocity measurement rather than the one obtained from the LIDAR device. On the other hand, due to the fact that some thrusters produce exactly the same or very similar torques, it is very hard to obtain a global isolation strategy based exclusively on angular velocity measurements. Therefore, the second isolation rule of the proposed global isolation strategy uses the information about the position dynamics contained in the fault detector's residual. This chronology of isolation steps gives to the fault an extra time to propagate into the translation dynamics.

\subsection{Thruster Group Isolation Using a Bank of NUIOs}

Let's consider the spacecraft as a rigid body (flex modes and slosh phenomena are not considered in this work), this model is given by (Sidi, 1997)

$$
\dot{\boldsymbol{\omega}}(t)=\boldsymbol{J}^{-1} \boldsymbol{B}_{T} \boldsymbol{u}_{f}(t)-\boldsymbol{J}^{-1} \boldsymbol{\omega}(t) \times \boldsymbol{J} \boldsymbol{\omega}(t)
$$

where $\boldsymbol{\omega}=[p, q, r]^{T}$ is the rotational velocity vector and $\boldsymbol{J} \in \mathbb{R}^{3 \times 3}$ is the real inertia matrix. In (13), both $\boldsymbol{\omega}$ and $\boldsymbol{J}$ are given in the chaser's body-fixed frame $\mathcal{F}_{b}$. Since the attitude model involves the inertia matrix $\boldsymbol{J}$ and its inverse $\boldsymbol{J}^{-1}$, robustness issue against uncertainties in $\boldsymbol{J}$ is a key feature in the design of the NUIO. This problem is addressed in the following subsection. 


\subsubsection{Chaser Attitude Dynamics and Inertia Uncertainty}

Let the inertia matrix $\boldsymbol{J}$ having the general form

$$
\boldsymbol{J}=\left[\begin{array}{lll}
J_{x x} & J_{x y} & J_{x z} \\
J_{x y} & J_{y y} & J_{y z} \\
J_{x z} & J_{y z} & J_{z z}
\end{array}\right]
$$

First, we define a factorization of $\boldsymbol{J}$ by introducing a diagonal matrix $\boldsymbol{J}_{d} \in \mathbb{R}^{9 \times 9}$ with the uncertain terms of $\boldsymbol{J}$, i.e.

$$
\boldsymbol{J}_{d}=\operatorname{diag}\left(J_{x x}, J_{y y}, J_{z z}, J_{x y} \boldsymbol{I}_{2}, J_{x z} \boldsymbol{I}_{2}, J_{y z} \boldsymbol{I}_{2}\right)
$$

where $\boldsymbol{I}_{2}$ is an identity matrix of size 2 . The $\boldsymbol{J}_{d}$ matrix can now be associated with two placement matrices $\boldsymbol{R}_{J}$ and $\boldsymbol{S}_{J}$,

$$
\boldsymbol{R}_{J}=\left[\begin{array}{lllllllll}
1 & 0 & 0 & 1 & 0 & 1 & 0 & 0 & 0 \\
0 & 1 & 0 & 0 & 1 & 0 & 0 & 1 & 0 \\
0 & 0 & 1 & 0 & 0 & 0 & 1 & 0 & 1
\end{array}\right], \quad \boldsymbol{S}_{J}^{T}=\left[\begin{array}{lllllllll}
1 & 0 & 0 & 0 & 1 & 0 & 1 & 0 & 0 \\
0 & 1 & 0 & 1 & 0 & 0 & 0 & 0 & 1 \\
0 & 0 & 1 & 0 & 0 & 1 & 0 & 1 & 0
\end{array}\right]
$$

to give the factorized expression of $\boldsymbol{J}$ as follows

$$
\boldsymbol{J}=\boldsymbol{R}_{J} \boldsymbol{J}_{d} \boldsymbol{S}_{J}
$$

The inertia uncertainty can be expressed by direct multiplicative uncertainty as

$$
\boldsymbol{J}_{d}=\boldsymbol{J}_{d 0}\left(\boldsymbol{I}+\boldsymbol{\Delta}_{J}\right)
$$

where $\boldsymbol{J}_{d 0}$ consists of nominal values of $\boldsymbol{J}_{d}$ and $\boldsymbol{\Delta}_{J}$ represents the uncertainty in the diagonal form

$$
\boldsymbol{\Delta}_{J}=\operatorname{diag}\left(\Delta J_{x x}, \Delta J_{y y}, \Delta J_{z z}, \Delta J_{x y} \boldsymbol{I}_{2}, \Delta J_{x z} \boldsymbol{I}_{2}, \Delta J_{y z} \boldsymbol{I}_{2}\right)
$$

with $\left|\Delta J_{i j}\right| \leq \bar{\delta}_{i j}, \forall i, j \in\{x, y, z\}$, where $0 \leq \bar{\delta}_{i j} \leq 1$ is the upper bound of the considered uncertainty level along the given axis. If $\bar{\delta}_{i j}<1$ for any $i, j$ couple, it is possible to reduce conservatism by introducing the following scaling

$$
\boldsymbol{\Delta}_{J}=\boldsymbol{W} \boldsymbol{\Delta}_{J}^{*}, \quad \boldsymbol{\Delta}_{J}^{* T} \boldsymbol{\Delta}_{J}^{*} \leq \boldsymbol{I}
$$

where

$$
\boldsymbol{W}=\operatorname{diag}\left(\bar{\delta}_{x x}, \bar{\delta}_{y y}, \bar{\delta}_{z z}, \bar{\delta}_{x y} \boldsymbol{I}_{2}, \bar{\delta}_{x z} \boldsymbol{I}_{2}, \bar{\delta}_{y z} \boldsymbol{I}_{2}\right)
$$

Finally, inserting (17) into (16) gives the inertia matrix expressed in the additive uncertainty form

$$
\boldsymbol{J}=\boldsymbol{J}_{0}+\boldsymbol{R}_{J}^{*} \Delta_{J}^{*} \boldsymbol{S}_{J}
$$

where $\boldsymbol{J}_{0}=\boldsymbol{R}_{J} \boldsymbol{J}_{d 0} \boldsymbol{S}_{J}$ and $\boldsymbol{R}_{J}^{*}=\boldsymbol{R}_{J} \boldsymbol{J}_{d 0} \boldsymbol{W}$. The inverse of $\boldsymbol{J}$ appears in (13), therefore, it is essential, to express this inverse in a factorized form. Proposition 1 provides a method to achieve it. 
Proposition 1 (Uncertain inertia inverse factorization). If $\left\|\boldsymbol{J}_{0}^{-1} \boldsymbol{R}_{J}^{*}\right\|\left\|\boldsymbol{S}_{J}\right\| \leq 1$, then the inverse of the uncertain inertia matrix (20) can be expressed as

$$
\boldsymbol{J}^{-1}=\boldsymbol{J}_{0}^{-1}+\boldsymbol{R}_{2} \boldsymbol{\Delta}_{2} \boldsymbol{S}_{2}
$$

where $\boldsymbol{R}_{2}, \boldsymbol{S}_{2}$ are constant matrices given by $\boldsymbol{R}_{2}=\boldsymbol{J}_{0}^{-1} \boldsymbol{R}_{J}^{*}\left\|\left(\boldsymbol{I}+\boldsymbol{S}_{J} \boldsymbol{J}_{0}^{-1} \boldsymbol{R}_{J}^{*}\right)^{-1}\right\|$ and $\boldsymbol{S}_{2}=$ $\boldsymbol{S}_{J} \boldsymbol{J}_{0}^{-1}$. Matrix $\boldsymbol{\Delta}_{2}$ satisfies $\boldsymbol{\Delta}_{2}^{T} \boldsymbol{\Delta}_{2} \leq \boldsymbol{I}$.

Proof: see Appendix A.

Utilizing the above proposition with the definition of the state vector $\boldsymbol{x}=\boldsymbol{\omega}$, it can be verified that equation (13) can be represented in the following nonlinear state space representation

$$
\begin{aligned}
& \dot{\boldsymbol{x}}(t)=\boldsymbol{A} \boldsymbol{x}(t)+\boldsymbol{\Phi}(\boldsymbol{x}(t))+\Delta \boldsymbol{\Phi}(\boldsymbol{x}(t))+(\boldsymbol{B}+\Delta \boldsymbol{B}) \boldsymbol{u}_{f}(t) \\
& \boldsymbol{y}(t)=\boldsymbol{C} \boldsymbol{x}(t)
\end{aligned}
$$

with the following assignments

$$
\begin{gathered}
\boldsymbol{\Phi}(\boldsymbol{x}(t))=-\boldsymbol{J}_{0}^{-1} \boldsymbol{x}(t) \times \boldsymbol{J}_{0} \boldsymbol{x}(t)-\boldsymbol{A} \boldsymbol{x}(t), \quad \Delta \boldsymbol{B}=\boldsymbol{R}_{2} \Delta_{2} \boldsymbol{S}_{2} \boldsymbol{B}_{T}, \quad \boldsymbol{A}=\left.\frac{\partial \dot{\boldsymbol{x}}}{\partial \boldsymbol{x}}\right|_{\left(x_{0}, J_{0}\right)} \\
\Delta \boldsymbol{\Phi}(\boldsymbol{x}(t))=-\boldsymbol{J}^{-1} \boldsymbol{x}(t) \times \boldsymbol{J} \boldsymbol{x}(t)+\boldsymbol{J}_{0}^{-1} \boldsymbol{x}(t) \times \boldsymbol{J}_{0} \boldsymbol{x}(t), \quad \boldsymbol{B}=\boldsymbol{J}_{0}^{-1} \boldsymbol{B}_{T}, \quad \boldsymbol{C}=\boldsymbol{I}
\end{gathered}
$$

This formulation is now suitable for the NUIO theory proposed in the subsection.

\subsubsection{Robust Nonlinear Unknown Input Observer Design}

Consider the model given by (22)-(23) without the nonlinear uncertainty $\Delta \boldsymbol{\Phi}(\boldsymbol{x}(t))$, but with a disturbance vector $\boldsymbol{d}$ occurring in the state equation (this will be justified later in Section 4.1.3), i.e.

$$
\begin{aligned}
& \dot{\boldsymbol{x}}(t)=\boldsymbol{A} \boldsymbol{x}(t)+\boldsymbol{\Phi}(\boldsymbol{x}(t))+(\boldsymbol{B}+\Delta \boldsymbol{B}) \boldsymbol{u}(t)+\boldsymbol{E} \boldsymbol{d}(t) \\
& \boldsymbol{y}(t)=\boldsymbol{C} \boldsymbol{x}(t)
\end{aligned}
$$

As usual in the UIO theory, the design of the observer parameters is done without fault consideration, i.e. $\boldsymbol{\Psi}=0 \Rightarrow \boldsymbol{u}_{\boldsymbol{f}}=\boldsymbol{u}$. Thus, fault sensitivity performance can only be checked a posteriori (see e.g. Patton et al. (2000)).

Assumption 1. It is assumed that $\boldsymbol{\Phi}(\boldsymbol{x})$ is Lipschitz in a region $\mathcal{S}$ containing the origin, i.e. $\left\|\boldsymbol{\Phi}\left(\boldsymbol{x}_{1}\right)-\boldsymbol{\Phi}\left(\boldsymbol{x}_{2}\right)\right\| \leq \gamma\left\|\boldsymbol{x}_{1}-\boldsymbol{x}_{2}\right\|, \quad \forall\left(\boldsymbol{x}_{1}, \boldsymbol{x}_{\mathbf{2}}\right) \in \mathcal{S}$ where $\gamma>0$ stands for the Lipschitz constant. If $\mathcal{S}=\mathbb{R}^{n}, \boldsymbol{\Phi}$ is globally Lipschitz. Otherwise, it is locally Lipschitz.

Assumption 2. It is assumed that $\boldsymbol{E}$ is of full column rank and that $\operatorname{rank}(\boldsymbol{C E})=\operatorname{rank}(\boldsymbol{E})$.

Note that Assumption 1 is reasonable in our case, since $\boldsymbol{\Phi}(\boldsymbol{x})$ in (22) is continuously differentiable on $\mathbb{R}^{3}$ and thus, it is locally Lipschitz. This means that the angular velocity shall be bounded in magnitude which is a reasonable assumption from a practical point of view, 
too. Assumption 2 can be done without loss of generality, see e.g. (Chen and Patton, 1999) if necessary.

Under Assumptions 1 and 2, the goal turns out to design the following NUIO

$$
\begin{aligned}
& \dot{\boldsymbol{z}}(t)=\boldsymbol{N} \boldsymbol{z}(t)+\boldsymbol{G u}(t)+\boldsymbol{L} \boldsymbol{y}(t)+\boldsymbol{M} \boldsymbol{\Phi}(\hat{\boldsymbol{x}}(t)) \\
& \hat{\boldsymbol{x}}(t)=\boldsymbol{z}(t)+\boldsymbol{H} \boldsymbol{y}(t)
\end{aligned}
$$

in such a way that $\hat{\boldsymbol{x}}$ lends robustness against the uncertainties $\Delta \boldsymbol{B} \boldsymbol{u}$ and is decoupled from the unknown inputs $\boldsymbol{d}$. In (27)-(28), $\hat{\boldsymbol{x}} \in \mathbb{R}^{n}$ stands for the estimate of $\boldsymbol{x}$ and $\boldsymbol{z} \in \mathbb{R}^{n}$ is an auxiliary signal. It can be verified that a solution to this problem yields if and only if

$$
\begin{aligned}
\boldsymbol{N} & =\boldsymbol{M A}-\boldsymbol{K} \boldsymbol{C}, \\
\boldsymbol{L} & =\boldsymbol{K}(\boldsymbol{I}-\boldsymbol{C H})+\boldsymbol{M A H}, \\
\boldsymbol{M} & =\boldsymbol{I}-\boldsymbol{H C}, \\
\boldsymbol{G} & =\boldsymbol{M B} \\
(\boldsymbol{I} & -\boldsymbol{H} \boldsymbol{C}) \boldsymbol{E}=\mathbf{0}
\end{aligned}
$$

The general solution to (33) can be written as

$$
\boldsymbol{H}=\boldsymbol{U}+\boldsymbol{Y} \boldsymbol{V}
$$

where $\boldsymbol{Y}$ must be chosen so that it does not cause rank deficiency of $\boldsymbol{H}$. Matrices $\boldsymbol{U}$ and $\boldsymbol{V}$ are given by

$$
\boldsymbol{U}=\boldsymbol{E}(\boldsymbol{C E})^{\dagger}, \quad \boldsymbol{V}=\boldsymbol{I}-(\boldsymbol{C E})(\boldsymbol{C E})^{\dagger}
$$

where $(\boldsymbol{C E})^{\dagger}$ denotes the generalized pseudo-inverse of the matrix $\boldsymbol{C E}$.

The aim is now to design the parameters $\boldsymbol{K}$ and $\boldsymbol{Y}$ such that the estimation error $\boldsymbol{e}=\boldsymbol{x}-\hat{\boldsymbol{x}}$ tends asymptotically to zero with maximum admissible Lipschitz constant $\gamma^{*}$ and such that the $\mathcal{L}_{2}$ gain from $\Delta \boldsymbol{B} \boldsymbol{u}$ to the estimation error $\boldsymbol{e}$ is bounded by

$$
\frac{\|\boldsymbol{e}\|_{\ell 2}}{\|\Delta \boldsymbol{B} \boldsymbol{u}\|_{\ell 2}} \leq \kappa, \quad \forall \boldsymbol{u} \in \mathcal{L}_{2}[0, \infty),\|\Delta \boldsymbol{B} \boldsymbol{u}\|_{\ell 2} \neq 0
$$

for a given $\kappa>0$. The following theorem provides a LMI-based method for NUIO design.

Theorem 1. Consider the (Lipschitz) nonlinear system given by (25)-(26). The NUIO given by (27)-(28) is asymptotically stable with maximum Lipschitz constant $\gamma^{*}$ and the $\mathcal{L}_{2}$ gain from $\Delta \boldsymbol{B u}$ to $\boldsymbol{e}$ is bounded by $\kappa>0$, if there exists a positive definite matrix $\boldsymbol{P}=\boldsymbol{P}^{T}>0$ and matrices $\overline{\boldsymbol{K}}, \overline{\boldsymbol{Y}}$ as solutions of the following optimization problem:

$$
\max _{P, \bar{K}, \bar{Y}} \xi
$$




$$
\text { s.t. }\left[\begin{array}{ccccc}
\boldsymbol{\Psi}_{11}+\boldsymbol{\Gamma}_{11} & \boldsymbol{\Omega}_{12} & \boldsymbol{\Omega}_{13} & \mathbf{0} & \mathbf{0} \\
* & -\boldsymbol{I} & \mathbf{0} & \mathbf{0} & \mathbf{0} \\
* & * & -\boldsymbol{I} & \mathbf{0} & \mathbf{0} \\
* & * & * & -\kappa^{2} \boldsymbol{I} & \boldsymbol{S}_{2} \boldsymbol{B}_{\boldsymbol{T}} \\
* & * & * & * & -\boldsymbol{I}
\end{array}\right]<0, \quad\left[\begin{array}{cc}
\xi & \gamma \\
* & 1
\end{array}\right] \geq 0
$$

where

$$
\begin{aligned}
& \boldsymbol{\Psi}_{11}=((\boldsymbol{I}-\boldsymbol{U} \boldsymbol{C}) \boldsymbol{A})^{T} \boldsymbol{P}+\boldsymbol{P}(\boldsymbol{I}-\boldsymbol{U} \boldsymbol{C}) \boldsymbol{A}+(1+\xi) \boldsymbol{I} \\
& \boldsymbol{\Gamma}_{11}=-(\boldsymbol{V} \boldsymbol{C} \boldsymbol{A})^{T} \overline{\boldsymbol{Y}}^{T}-\overline{\boldsymbol{Y}} \boldsymbol{V} \boldsymbol{C} \boldsymbol{A}-\boldsymbol{C}^{T} \overline{\boldsymbol{K}}^{T}-\overline{\boldsymbol{K}} \boldsymbol{C} \\
& \boldsymbol{\Omega}_{12}=\boldsymbol{P}(\boldsymbol{I}-\boldsymbol{U} \boldsymbol{C})-\overline{\boldsymbol{Y}} \boldsymbol{V} \boldsymbol{C} \\
& \boldsymbol{\Omega}_{13}=\boldsymbol{P}(\boldsymbol{I}-\boldsymbol{U} \boldsymbol{C}) \boldsymbol{R}_{2}-\overline{\boldsymbol{Y}} \boldsymbol{V} \boldsymbol{C} \boldsymbol{R}_{2}
\end{aligned}
$$

Once the problem is solved, then

$$
\boldsymbol{K}=\boldsymbol{P}^{-1} \overline{\boldsymbol{K}}, \quad \boldsymbol{Y}=\boldsymbol{P}^{-1} \overline{\boldsymbol{Y}}, \quad \gamma^{*}=\sqrt{\xi}
$$

Proof: see Appendix B.

Remark 1. It should be outlined that NUIO designed according to Theorem 1 tolerates any additive uncertainty $\boldsymbol{\Delta} \boldsymbol{\Phi}(\boldsymbol{x})$ in $\boldsymbol{\Phi}_{\Delta}(\boldsymbol{x})$, i.e $\boldsymbol{\Phi}_{\Delta}(\boldsymbol{x})=\boldsymbol{\Phi}(\boldsymbol{x})+\boldsymbol{\Delta}(\boldsymbol{x})$, with Lipschitz constant less than or equal to $\gamma^{*}-\gamma$, see the work of Abbaszadeh and Marquez (2009) for a discussion.

Remark 2. The maximization of the admissible Lipschitz constant $\gamma^{*}$ may result in unsatisfactory dynamical behaviour of the state estimation error. To overcome this problem, the $\mathcal{D}$-stability concept proposed by Chilali and Gahinet (1996) can be used jointly with Theorem 1, thanks to the LMI formulation (38). Substituting (31), (34) and (43) into (29) and transposing, it yields $\boldsymbol{N}^{T}=\boldsymbol{A}^{T}-(\boldsymbol{U} \boldsymbol{C} \boldsymbol{A})^{T}-(\overline{\boldsymbol{Y}} \boldsymbol{V} \boldsymbol{C A})^{T} \boldsymbol{P}^{-1}-(\overline{\boldsymbol{K}} \boldsymbol{C})^{T} \boldsymbol{P}^{-1}$. Then, direct application of the developments proposed in (Chilali and Gahinet, 1996) shows that the eigenvalues of $\boldsymbol{N}$ can be assigned into a prescribed region $\mathcal{D}=\cap_{k=1}^{n_{s}} \mathcal{D}_{k}$ if there exist a common Lyapunov matrix $\boldsymbol{P}=\boldsymbol{P}^{T}>0$ and matrices $\overline{\boldsymbol{K}}$ and $\overline{\boldsymbol{Y}}$ such that the set of $n_{s}$ LMIs

$$
\begin{gathered}
\boldsymbol{\alpha}_{k} \otimes \boldsymbol{P}+\boldsymbol{\beta}_{k} \otimes\left(\boldsymbol{A}^{T} \boldsymbol{P}-(\boldsymbol{U} \boldsymbol{C A})^{T} \boldsymbol{P}-(\overline{\boldsymbol{Y}} \boldsymbol{V} \boldsymbol{C} \boldsymbol{A})^{T}-(\overline{\boldsymbol{K}} \boldsymbol{C})^{T}\right)+ \\
\boldsymbol{\beta}_{k}^{T} \otimes(\boldsymbol{P} \boldsymbol{A}-\boldsymbol{P}(\boldsymbol{U} \boldsymbol{A})-\overline{\boldsymbol{Y}} \boldsymbol{V} \boldsymbol{C}-\overline{\boldsymbol{K}} \boldsymbol{C})<0 \quad k=1,2, \ldots, n_{s}
\end{gathered}
$$

is simultaneously satisfied. In this expression, $\boldsymbol{\alpha}_{k}$ and $\boldsymbol{\beta}_{k}$ are matrices of appropriate dimension defining each region $\mathcal{D}_{k}$.

\subsubsection{Comments on Computational Issues}

The Lipschitz constant $\gamma$ for $\boldsymbol{\Phi}(\boldsymbol{\omega})$ can be easily computed using a constrained optimization algorithm over the set $\mathcal{S}_{\omega}=\left\{\boldsymbol{\omega} \in \mathbb{R}^{3}:\left|\omega_{k}\right| \leq \bar{\omega}, k=1,2,3\right\}$, where $\bar{\omega}$ is the upper bound of the angular velocity for each axis. The LMI region assignment approach described in Remark 2 is also considered to adjust adequately the dynamics of the NUIOs. For each NUIO, the chosen region $\mathcal{D}$ results in the intersection of three elementary LMI regions $\mathcal{D}_{k}, k=1,2,3$ defined according to: 
- $\mathcal{D}_{1}$ : left-half plane delimited by a vertical line $-\alpha, \alpha>0$;

- $\mathcal{D}_{2}$ : disk with center at $(-b, 0)$ and radius $c$;

- $\mathcal{D}_{3}$ : conic region with center at the origin and inner angle $0<\beta<\pi / 2$ pointing left.

These parameters $(\alpha, b, c, \beta)$ have to be tuned such that the estimation error dynamics react quick enough to any type of considered fault, allowing early distinction among the healthy/faulty thruster groups $\mathcal{S}_{T k}, k=1, \ldots, 5$. (See the following section about the proposed thruster group isolation strategy.)

For each thruster group $\mathcal{S}_{T k}, k=1, \ldots, 5$ (see equation (4) for definition), a dedicated NUIO is designed based on Algorithm 1. The $k^{\text {th }}$ NUIO is such that it can fully estimate the angular velocity $\boldsymbol{\omega}$ with all control inputs except those associated with $\mathcal{S}_{T k}$, i.e. with $u_{i}, \forall i \in \mathcal{S}_{a l l} \backslash \mathcal{S}_{T k}$. On the other hand, $\boldsymbol{d}$ in equation (25) stays for the control inputs associated with $\mathcal{S}_{T k}$ (i.e. $\left.u_{i}, \forall i \in \mathcal{S}_{T k}\right)$. As a result, the NUIO dedicated to the group $\mathcal{S}_{T k}$ shall not be affected by faults occurring in the thrusters belonging to $\mathcal{S}_{T k}$ due to the decoupling property, while all the other NUIOs will be ("are expected to be" to be more precise since the design of the NUIOs are done without fault sensitivity constraint).

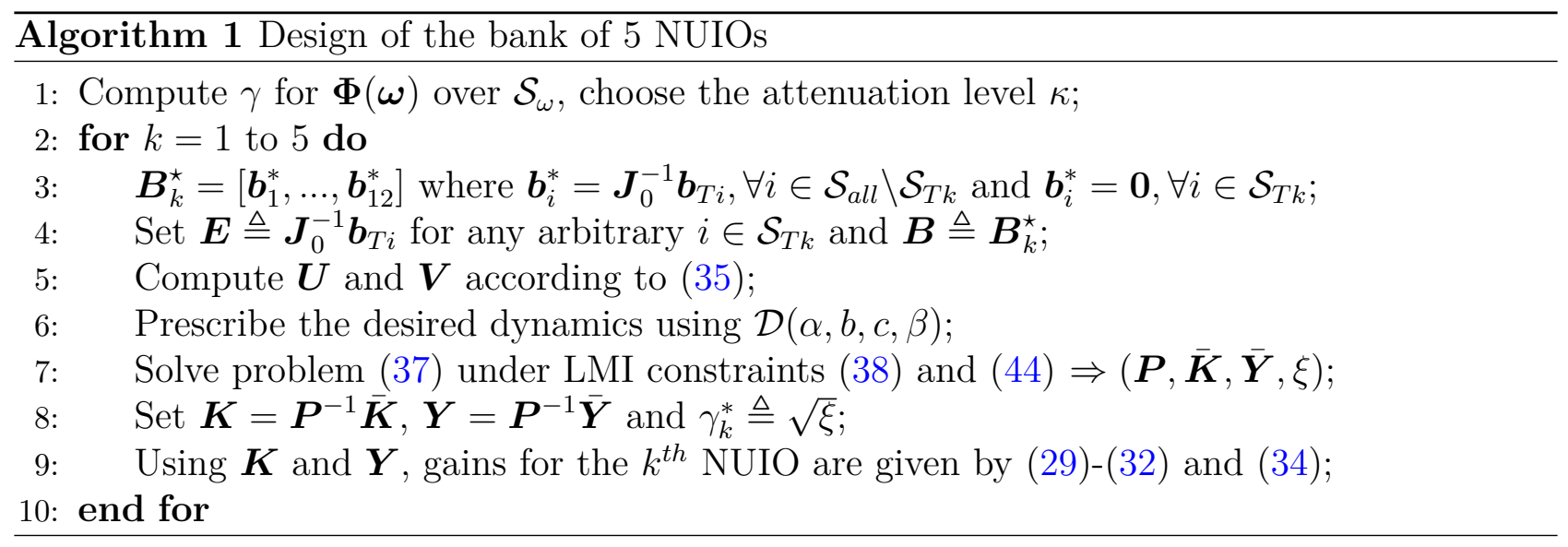

It is important to note that $\boldsymbol{d}$ can be exactly decoupled only if the columns of $\boldsymbol{\Delta} \boldsymbol{B}$ related to $\boldsymbol{d}$ are zero. If this is not the case, only the known directions, i.e. $\boldsymbol{b}_{i}^{*}=\boldsymbol{J}_{0}^{-1} \boldsymbol{b}_{T i}, i \in \mathcal{S}_{T k}$, can be exactly decoupled, while the uncertain columns $\Delta \boldsymbol{b}_{i}^{*}, i \in \mathcal{S}_{T k}$ (columns of $\Delta \boldsymbol{B}$ associated with $\mathcal{S}_{T k}$ ) are attenuated in $\mathcal{L}_{2}$ sense (with upper bound $\kappa$ ) since the entire $\Delta \boldsymbol{B}$ matrix is considered in (36). Furthermore, if a constant $\gamma^{*}$ linked to a given NUIO verifies $\gamma^{*}>\gamma$, then the associated observer tolerates an additionally nonlinear uncertainty in $\Phi_{\Delta}(\boldsymbol{\omega})$, see Remark 1.

Note that all observers estimate only the angular rate $\boldsymbol{\omega}$ of the chaser. Therefore, the computational burden is reduced since there is no need to process the entire state vector (i.e., the linear position/velocity and the attitude in addition). For real-time reasons, the bank of 5 NUIOs is triggered only when the decision signal $\varrho^{J_{t h}}$ indicates the fault occurrence, i.e., when $\varrho^{J_{t h}}(t)=1$ for $t \geq t_{d}$. Even if only $\boldsymbol{\omega}$ is estimated, keeping the NUIOs switched off before the fault is detected seems to be a good strategy, concerning the nonlinear nature of 
the observer. Each observer is initialized then with the known measurement at time $t_{d}$, i.e., $\hat{\boldsymbol{\omega}}_{k}\left(t_{d}\right)=\boldsymbol{\omega}\left(t_{d}\right), \forall k \in\{1, \ldots, 5\}$. By this, all observers have a zero initial estimation error. Hence, the observer initial convergence (transient phase) problem is avoided.

\subsubsection{Thruster Group Isolation Logic - First Stage}

Due to the aforementioned structuration of the bank of the NUIOs, it seems clear that the NUIO with the minimum estimation error (in some norm sense) reveals that a fault occurs in the associated set $\mathcal{S}_{T k}$. Such a property provides an efficient isolation rule that can be written according to

$$
\bar{\sigma}_{g}(t)=\underset{k}{\arg \min }\left\|\boldsymbol{e}_{k}(t)\right\|, \quad t>t_{d}
$$

where $\boldsymbol{e}_{k}(t)$ denotes the estimation error at time $t$ associated with the $k^{t h}$ NUIO. Note that the bank of NUIOs is triggered only when the fault indicating signal $\varrho^{J_{t h}}$ (see Eq. (11)) indicates that a fault has occurred, that is for $t>t_{d}$. To avoid initial transition phenomena and to ensure robustness against noise, a confirmation time window, $\delta_{g}>0$, is introduced, i.e.

$$
t_{g}=\underset{t \geq t_{d}+\delta_{g}}{\arg \inf }\left\{\bar{\sigma}_{g}(t)=\bar{\sigma}_{g}(\vartheta), \forall \vartheta \in\left(t-\delta_{g}, t\right]\right\}
$$

where $t_{g}$ is the isolation time of the faulty thruster group $j=\bar{\sigma}_{g}\left(t_{g}\right)$.

In ideal conditions, at this isolation stage, the minimum time $\left(t_{d}-t_{f}\right)+\delta_{g}$ has elapsed from the fault occurrence at $t=t_{f}$, thus allowing extra time for the fault to induce observable dynamic deviations in the translation dynamics contained in the residual signal $\boldsymbol{r}$ given by (10). Therefore, as soon as the faulty thruster group index " $j$ " is confirmed, the faulty thruster can be uniquely isolated by simply examining the degree of alignment between $\boldsymbol{r}$ and the fixed force vector directions $\boldsymbol{b}_{F k}, k \in \mathcal{S}_{T j}$ (see equation (3) for definition of $\boldsymbol{b}_{F k}$ ) under the assumption that the fault type is known. This is the purpose of the next subsection.

\subsection{Final Thruster Fault Isolation - Second Stage}

As soon as the faulty thruster group $\mathcal{S}_{T j}$ is identified at the first stage, the faulty thruster can be easily isolated by examining the angle of the vector $\boldsymbol{r}$ along the fixed force directions $\boldsymbol{b}_{F k}, \forall k \in \mathcal{S}_{T j}$. If the $k^{t h}$ thruster is faulty, then vectors $\boldsymbol{r} \in \mathbb{R}^{3}$ and $\boldsymbol{b}_{F k} \in \mathbb{R}^{3}$ should be collinear (owing the fault model (7)). The degree of collinearity can be computed using the direction cosine approach: $\theta_{d}^{k}=\boldsymbol{b}_{F k} \cdot \boldsymbol{r} /\left(\left\|\boldsymbol{b}_{F k}\right\|\|\boldsymbol{r}\|\right)$, where $\theta_{d}^{k}$ is the angle between the vectors $\boldsymbol{r}$ and $\boldsymbol{b}_{F k}$. If $\boldsymbol{r}$ and $\boldsymbol{b}_{F k}$ are collinear, then $\cos \left(\theta_{d}^{k}\right)=1$. Thus, the following rule is proposed to isolate the faulty thruster uniquely:

$$
\bar{\sigma}(t)=\underset{k \in \mathcal{S}_{T j}}{\arg \min }\left(\rho(t) \frac{\boldsymbol{b}_{F k} \cdot \boldsymbol{r}(t)}{\left\|\boldsymbol{b}_{F k}\right\|\|\boldsymbol{r}(t)\|}\right), \quad t \geq t_{g}
$$

In this equation, $\rho$ determines whether an "open-" or "closed-type" thruster fault has occurred (see Section 2.1 about fault considerations). The notation $t \geq t_{g}$ indicates that this rule is applied only when the NUIO-based strategy (first stage) subscribed and confirmed the fault to the subset $\mathcal{S}_{T j}$. 
With respect to $\rho$, the following two definitions are adopted depending on the identified thruster group $\mathcal{S}_{T j}$, i.e.

a) Definition for $j=1, \ldots, 4$

Recalling the geometrical properties in terms of torque directions (see Section 2), i.e. that thrusters belonging to the first four groups $\mathcal{S}_{T j}, j=1, \ldots, 4$ generate torques in the same direction within these groups, i.e. $\boldsymbol{b}_{T k}=\boldsymbol{b}_{T h}, \forall k, h \in \mathcal{S}_{T j}$. This property allows to consider the following definition for $\rho$ when $j \neq 5$, i.e.

$$
\rho^{(1: 4)}(t)=\operatorname{sign}\left(\boldsymbol{b}_{T k} \cdot \hat{\boldsymbol{T}}_{\text {bias }}(t)\right), \quad \text { for any } k \in \mathcal{S}_{T j}, \quad j \neq 5
$$

where $\hat{\boldsymbol{T}}_{\text {bias }} \in \mathbb{R}^{3}$ is the estimate of the real torque bias $\boldsymbol{T}_{\text {bias }}$ and $\operatorname{sign}(\cdot)$ stands for the signum function. This bias is due to the faulty thruster (see equation (7)) and should be understood as follows ${ }^{5}$

$$
\boldsymbol{T}_{\text {bias }}(t)=-\boldsymbol{B}_{T} \boldsymbol{\Psi}(t) \boldsymbol{u}(t), \quad \boldsymbol{\Psi}(t) \neq \mathbf{0}
$$

It is obvious that the two fault types, i.e. "open-" and "closed-type", result in exactly opposite torque bias (shift) relative to the torque direction $\boldsymbol{b}_{T k}, \forall k \in \mathcal{S}_{T j}, j \neq 5$.

The torque bias (49) can be estimated using an EKF based on the nominal $\left(\boldsymbol{J} \triangleq \boldsymbol{J}_{0}\right)$ attitude dynamics model (13), see for instance (Posch et al., 2013) for realisation details. Note that in (48), the direction vector $\boldsymbol{b}_{T k}$ can be any from $\mathcal{S}_{T j}$ since they are equal for all $j=1, \ldots, 4$.

\section{b) Definition for $j=5$}

Considering the thruster group 5 , it is obvious that the previous strategy cannot be used since $\boldsymbol{b}_{T k}, k \in \mathcal{S}_{T 5}$ are not unique/same-valued direction vectors, see equation (6). However, a special property of thrusters belonging to this subset is that they barely produce any torque in the $\mathrm{x}$ - and $\mathrm{y}$-axis. This enables to focus only on the z-axis. Thus, the following definition for $\rho$ when $j=5$ is proposed:

$$
\rho^{(5)}(t)=f_{W a l d}\left(r_{\text {bias }}\left(t_{k}\right)\right), \quad j=5
$$

where $r_{\text {bias }}\left(t_{k}\right)=\hat{T}_{\text {bias }}^{z}\left(t_{k}\right)-\hat{T}_{\text {bias }}^{z}\left(t_{k-1}\right), \hat{T}_{\text {bias }}^{z}$ is the third component (i.e. the component on the z-axis) of $\hat{\boldsymbol{T}}_{\text {bias }}$ and $f_{\text {Wald }}(\cdot)$ stands for the sequential Wald test for the variance applied on $r_{\text {bias }}$. This test can result in three possible situations:

$$
f_{\text {Wald }}\left(r_{\text {bias }}\left(t_{k}\right)\right)=\left\{\begin{aligned}
1 & \text { if decision in favour of "closed-type" } \\
0 & \text { if no decision has been adopted } \\
-1 & \text { if no decision in favour of "open-type" }
\end{aligned}\right.
$$

Implementation details on the sequential Wald test, also known as Sequential Probability Ratio Test (SPRT) test, can be found in (Basseville and Nikiforov, 1993).

\footnotetext{
${ }^{5}$ In other words, this bias can be also understood as a difference (bias) between the real torques applied on the spacecraft and the torques as seen from the controller point of view.
} 
Improvement of the Strategy

For the thruster group number 5, taking into account (6), it is possible to slightly improve the reliability of the isolation algorithm (47) by dividing the set $\mathcal{S}_{T 5}$ into two smaller subsets, i.e. $\mathcal{S}_{T 5}^{a}=\{3,12\}$ and $\mathcal{S}_{T 5}^{b}=\{6,9\}$. Now, the isolation rule $(47)$ can be redefined for $j=5$ as follows

$$
\bar{\sigma}(t)=\left\{\begin{array}{cc}
\underset{k \in \mathcal{S}_{T 5}^{a}}{\arg \min }\left(\rho^{(5)}(t) \frac{\boldsymbol{b}_{F k} \cdot \boldsymbol{r}(t)}{\left\|\boldsymbol{b}_{F k}\right\|\|\boldsymbol{r}(t)\|}\right), & \text { if } \min _{k \in \mathcal{S}_{T 5}^{a}} \rho^{(5)}\left(\boldsymbol{b}_{T k} \cdot \hat{\boldsymbol{T}}_{\text {bias }}\right) \geq \min _{k \in \mathcal{S}_{T 5}^{b}} \rho^{(5)}\left(\boldsymbol{b}_{T k} \cdot \hat{\boldsymbol{T}}_{\text {bias }}\right) \\
\underset{k \in \mathcal{S}_{T 5}^{b}}{\arg \min }\left(\rho^{(5)}(t) \frac{\boldsymbol{b}_{F k} \cdot \boldsymbol{r}(t)}{\left\|\boldsymbol{b}_{F k}\right\|\|\boldsymbol{r}(t)\|}\right), & \text { otherwise }
\end{array}\right.
$$

Now, the logic (47) is able to isolate any of the four considered fault scenarios (see Section 2.1), thus thruster fault of both types, within any truster group $\mathcal{S}_{T j}, j=1, \ldots, 5$ ( $\operatorname{supposing}$ that the thruster group isolation $j=\bar{\sigma}_{g}$ was successful).

Finally, another confirmation window, $\delta>0$, is introduced according to

$$
t_{i}=\underset{t \geq t_{g}+\delta}{\arg \inf }\{\bar{\sigma}(t)=\bar{\sigma}(\vartheta), \forall \vartheta \in(t-\delta, t]\}
$$

where $t_{i}$ is the isolation time of the faulty thruster. Let $i=\bar{\sigma}\left(t_{i}\right)$ for future reference.

\section{Fault Accommodation}

Once a faulty thruster is isolated, a fault accommodation mechanism has to be engaged in order to maintain the capture objectives of the MSR mission. To carry out such objectives, TAS has designed the thruster configuration presented in Section 2. This configuration disposes of some Degrees of Freedom (DoF) to achieve fault tolerance (functional redundancy). Particularly, the set of $N=12$ thrusters is placed on the chaser spacecraft (see Fig. 1) such that the nominally attainable set $\mathcal{W}_{a}$ of propulsion moments $\boldsymbol{T}$ and forces $\boldsymbol{F}$ is relatively close to the sets obtained by combining the thrust of any $N-1=11$ thruster. From a practical viewpoint it means that it is possible to achieve the required capture accuracy and the necessary GNC performance with only eleven healthy thrusters. On the other hand, the nominal 6 DoF control law that is planned to be implemented on-board, is designed to guarantee the capture objectives such as: attitude alignment versus the target, the longitudinal and lateral velocities and the position in the rendezvous corridor. Since the CA technique do not require any modification in the control law, it motivates to propose the fault tolerance solution to be based on this philosophy. Moreover, the CA solution is further justified by the fact that all thrusters are individually equipped with a Thruster Latch Valve (TLV) able to disengage the propellant arrival, switching off de facto the associated thruster. Thus, as soon as the $i^{\text {th }}$ thruster is confirmed to be faulty by $\bar{\sigma}$, see (47) and (52), the faulty thruster is switched off using the dedicated TLV and the desired forces $\boldsymbol{F}_{d}$ and torques $\boldsymbol{T}_{d}$ of the controller are redistributed among the remaining $N-1$ healthy thrusters. Figure 3 gives an overview of the proposed FDI/CA-based FTC solution implemented within the GNC architecture. 


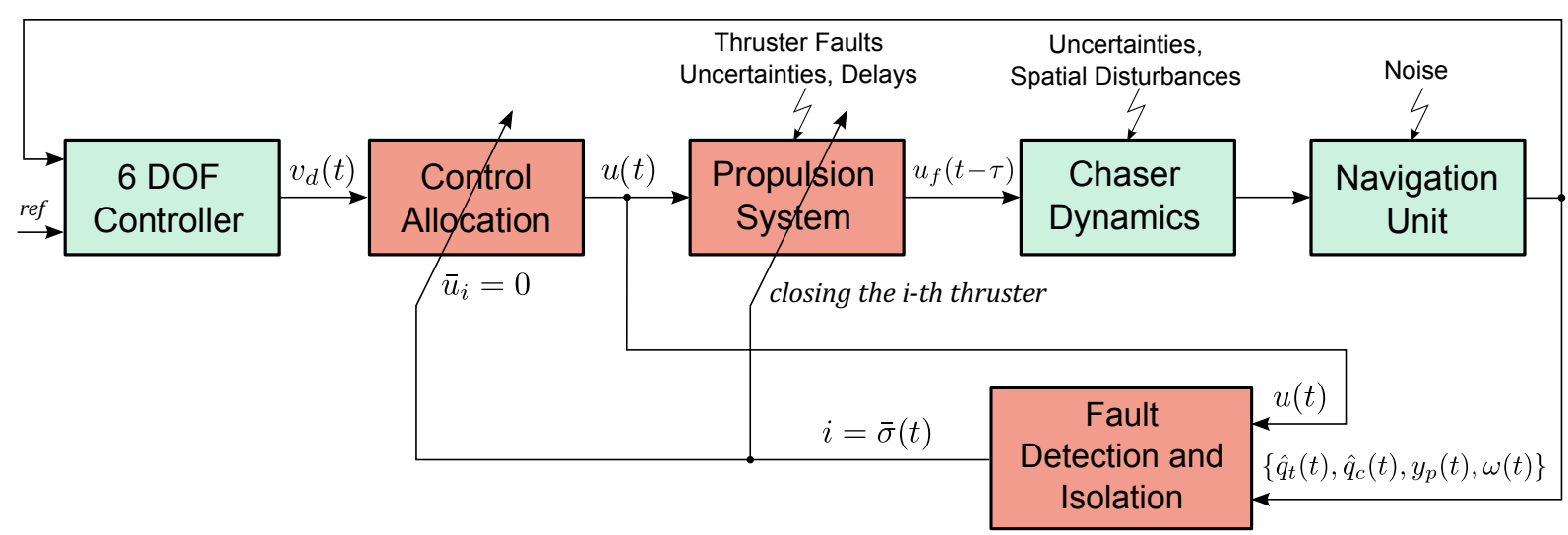

Figure 3: FDI/CA-based FTC strategy for thruster faults implemented within the GNC architecture

\subsection{Reconfigurable Control Allocation}

The on-board CA algorithm shall determine in real-time, i.e. at each control cycle (10 $\mathrm{Hz}$ frequency), the proper thruster selection and their firing times to achieve the controllercommanded torque and force impulses. Many CA algorithms have potential to be applied, see (Johansen and Fossen, 2013) for a recent survey on CA techniques. To make use of the remaining healthy thrusters in case of a failure, it is required to reconfigure the CA scheme (re-allocation). This re-allocation can be achieved easily by changing some constraints or parameters of the existing CA algorithms.

In this paper, a modified version of the NIPC approach is proposed. The original version of the NIPC algorithm was presented by Jin et al. (1995). The NIPC method solves the following optimization problem

$$
\begin{gathered}
\boldsymbol{u}=\underset{\boldsymbol{u}}{\arg \min }\left\|\boldsymbol{W}_{v}\left(\bar{B} \boldsymbol{u}-\boldsymbol{v}_{d}\right)\right\|_{p} \\
\text { s.t. } 0 \leq u_{k} \leq \bar{u}_{k}, \forall k \in \mathcal{S}_{\text {all }}
\end{gathered}
$$

where $\overline{\boldsymbol{B}}=\left[\overline{\boldsymbol{b}}_{1}, \ldots, \overline{\boldsymbol{b}}_{12}\right]=\left[\boldsymbol{B}_{T}^{T} \boldsymbol{B}_{F}^{T}\right]^{T}$ is the overall thruster configuration matrix, $\boldsymbol{v}_{d}=\left[\boldsymbol{T}_{d}^{T} \boldsymbol{F}_{d}^{T}\right]^{T}$ is the vector of the desired torque and force commands of the 6 DoF control law synthesized by the 6 DoF controller and followed by the thruster modulator unit, and $\bar{u}_{k}$ is the maximum opening duration of the $k^{\text {th }}$ thruster. The core of the fault tolerance principle is that if the $i^{\text {th }}$ thruster is faulty, then $\bar{u}_{i}$ is set to 0 . The weighting matrix $\boldsymbol{W}_{v}$ affects the prioritization among torque/force components when $\bar{B} \boldsymbol{u}-\boldsymbol{v}_{d}$ cannot be attained due to thruster physical constraints. The different choices of the vector $p$-norm in (53) result in:

1. Minimum flow rate allocation: $\min \|\boldsymbol{u}\|_{1}$

2. Minimum power allocation: $\min \|\boldsymbol{u}\|_{2}$

3. Minimum peak torque/force allocation: $\min \|\boldsymbol{u}\|_{\infty}$ 
Using the minimum flow rate allocation will yield the greatest control authority for flow rate limited thruster systems. Similarly for the other two allocations. It is known that stability of the closed-loop system can be guaranteed as long as the constraints of the optimization problem (53) are met (feasibility implies stability).

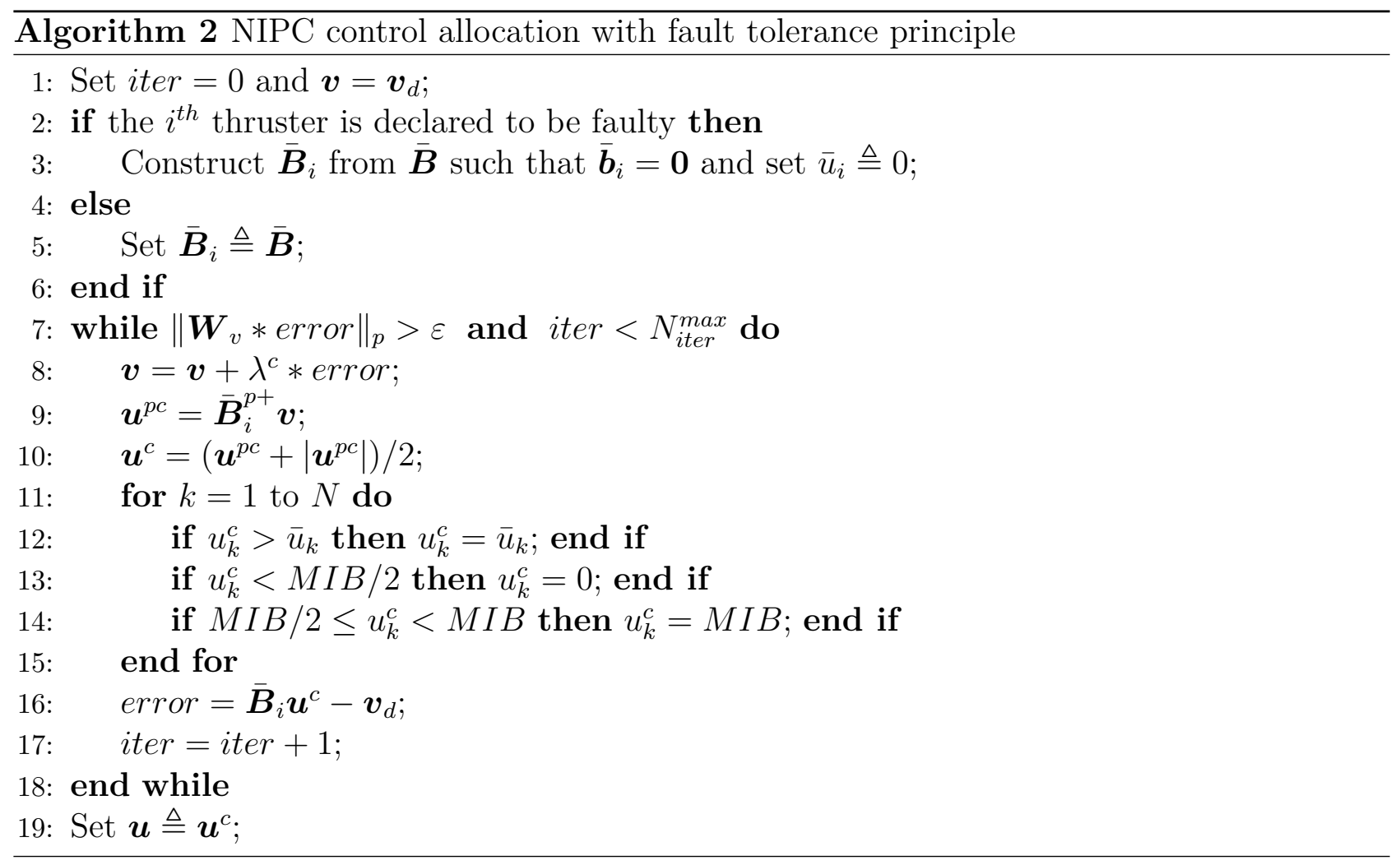

The proposed NIPC method that solves the re-allocation problem to ensure thruster fault tolerance, is given in Algorithm 2. This algorithm also solves the optimization problem (53). It terminates if a certain precision $\varepsilon \geq 0$ of the allocated torques/forces, weighted by $\boldsymbol{W}_{v}$, is achieved (typical choice is $\varepsilon \rightarrow 0$ ) or if the maximum number of iterations $N_{i t e r}^{\max }$ is reached. $N_{i t e r}^{m a x}$ can be considered to reflect the max computation time available. In Algorithm 2, MIB stands for the Minimum Impulse Bit, i.e. the minimum shooting time that a thruster can execute, $\lambda^{c}>0$ allows to manage the convergence time of the algorithm and $\overline{\boldsymbol{B}}_{i}^{p+}$ stands for the generalized inverse of $\overline{\boldsymbol{B}}_{i}$ given in step $\mathbf{3}$ (optimal in the sense of the chosen p-norm). It is obvious, that both $N_{i t e r}^{\max }$ and $\lambda^{c}$ influence the computational burdens of the algorithm.

Fault tolerance is achieved due to step 3 and consequently to steps 9 and $\mathbf{1 2}$ in the Algorithm 2. The index " $i$ " being determined by the FDI unit. Changing the minimization objective in (53) is very simple since it results in changing the criterion $p \in\{1,2, \infty\}$ in steps $\mathbf{7}$ and $\mathbf{9}$.

Remark 3. The NIPC algorithm has been compared with other powerful CA approaches presented in (Härkegård, 2003). Results from a numerical campaign have shown that the NIPC approach constitutes a good trade-off between accuracy and computational complexity. This is 
mostly due to the algorithm's conceptual simplicity, i.e. the matrices $\overline{\boldsymbol{B}}_{i}^{p+}$ in step $\mathbf{9}$ are all fixed, thus it is possible to pre-compute them all off-line. This enables to reduce the computational burdens, but the price to pay is a higher memory consumption.

\section{Simulation Campaign}

The scenario considered in this study is focused on the terminal rendezvous phase, which brings the chaser from approximately $20 \mathrm{~m}$ range up to the capture point. The objective is to successfully capture the target. To achieve this, the MSR capture conditions in terms of positions and velocities, and of relative attitude and angular rates must be achieved within a certain precision (see Table 1 for numerical values). Furthermore, during the whole rendezvous phase, the chaser spacecraft must maintain its position within the rendezvous corridor and must keep its attitude pointing towards the target with a maximum misalignment of 20 degrees on all the axis (roll, pitch, and yaw axes).

\begin{tabular}{lllll} 
& Capture condition & Nominal value & Max variation & Unit \\
\hline \hline \multirow{2}{*}{ Translational } & Position misalignment on +X face & 0.0 & 0.20 & $\mathrm{~m}$ \\
conditions & Longitudinal X velocity accuracy & 0.1 & 0.05 & $\mathrm{~m} / \mathrm{s}$ \\
& Lateral Y and Z velocity error & 0.0 & 0.04 & $\mathrm{~m} / \mathrm{s}$ \\
\hline Rotational & Angular rate error & 0 & $0.3^{\star}$ & $\mathrm{deg} / \mathrm{s}$ \\
conditions & Angular misalignment & 0 & $2^{\star}$ & $\mathrm{deg}$ \\
\hline \hline
\end{tabular}

Table 1: Baseline MSR conditions for successful capture ( ${ }^{\star}$ are $3 \sigma$ requirements)

The FTC strategy described in the previous sections has been implemented within the MSR high-fidelity industrial simulator provided by Thales Alenia Space industries. This simulator includes a nonlinear model of the rigid body dynamics of the chaser and target in a Mars orbit. Simulation assumes that Mars is in a Keplerian orbit about the Sun. The chaser and target orbits around Mars are modelled using Gauss' equations, with the gravitational field of Mars calculated using a spherical harmonic expansion with the Mars50c coefficients (Konopliv and Sjogren, 1995; Hartley et al., 2012). The attitude dynamics are modelled assuming that the chaser and target are rigid bodies (Sidi, 1997).

Following the design steps given in Algorithm 1, a bank of 5 NUIOs has been designed. The numerical values for $\alpha, b, c, \beta$, and $\kappa$ being fixed to $0,0.18,0.05, \pi / 4$, and 0.9 for all NUIOs, respectively. The numerical values of $\gamma$ and $\gamma^{*}$ are found to be 0.9047 and $1.4039 \times 10^{4}$. The selected parameters for the NIPC (see Algorithm 2) algorithm correspond to: $\boldsymbol{W}_{v}=\boldsymbol{I}$, $N_{\text {iter }}^{\max }=350, \lambda^{c}=1.89, \varepsilon=10^{-7}$ and $p=2$, i.e. the $2^{\text {nd }}$ vector norm was chosen leading to minimum power allocation. Each thruster is considered to have $M I B=0.068 \mathrm{~s}$. Above this, the actual commanded open durations are quantised by step of $0.01 \mathrm{~s}$. The GLR decision test given by (11) has been implemented recursively with $J_{t h}=33, T_{s}=0.1 \mathrm{~s}, t_{0}=100 \mathrm{~s}$ and $w_{i}=1 / 3, \forall i \in\{1,2,3\}$. The chosen threshold $J_{t h}$ has been determined through Monte Carlo simulations to ensure minimum (ideally zero) false alarm rate. This approach is widely used 
in the FDI community (see Patton et al. (2006) for more details). For the two-stage isolation logic, a confirmation window $\delta_{g}=1.5 \mathrm{~s}$ has been considered in (46) and $\delta=0.5 \mathrm{~s}$ in (52). The $4^{\text {th }}$ order Runge-Kutta integration method has been used to propagate the nonlinear equations for the EKF to obtain the estimate $\hat{\boldsymbol{T}}_{\text {bias }}$ of the torque bias. The EKF state covariance matrix was tuned such that the estimated torque bias "directions" are as close as possible to the real ones. The measurement covariance matrix has been selected based on the knowledge of the gyro model.

Figure 4 serves as a simulation example and aims to highlight the need for an active FTC solution. This example corresponds to a fully open thruster fault (i.e. case 1) occurring at $t_{f}=1100 \mathrm{~s}$ and affecting thruster No.7. To emphasize the relevance of the engagement of the proposed FTC scheme into the GNC system, two identical simulations are carried out. First, when the proposed FTC strategy is active (FTC on), and second, when it is disengaged (FTC off). Figure 4 clearly illustrates the consequence when such a fault is not accommodated, i.e.

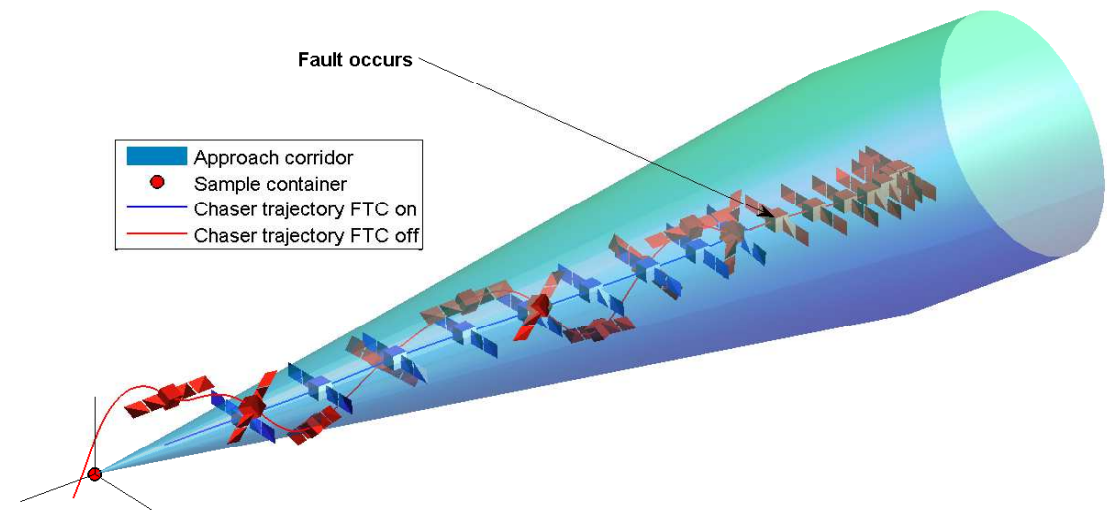

Figure 4: Chaser trajectory within the MSR rendezvous corridor

the chaser misses the target and the mission fails. On the other hand, when the proposed approach is engaged, the chaser maintains nominal trajectory, i.e. stays inside the rendezvous corridor and the MSR capture requirements are met. Furthermore, it can be inferred from Fig. 4 that the chaser keeps its attitude pointing towards the target all the time.

A Monte Carlo simulation campaign is often used in the industry to test and validate the performance of an FDI/FTC system. In this simulation study, a high number of simulation models with randomly drawn dynamics is associated with the following three thruster fault scenarios:

- case 1: fully open thruster, i.e. $m_{\text {leak }}=1$;

- case 2: bipropellant leakage ranging from $7 \%$ to $20 \%$, i.e. $m_{\text {leak }} \sim \mathcal{U}(0.07,0.2)$;

- case 3: loss of efficiency ranging from $30 \%$ to $100 \%$, i.e. $m_{\text {loss }} \sim \mathcal{U}(0.3,1)$.

The selected leakage and efficiency loss intervals were determined based on the study presented in (Fonod et al., 2014b). In this study, it was shown that if the FDI unit fails to detect or 
isolate a small thruster fault (e.g. $m_{\text {loss }} \lesssim 15 \%$ ), the effect that this fault has on the GNC system and/or on the final MSR capture performance requirements is negligible. It is due to the fact that such relatively small fault has a very little impact on the system dynamics and shall be compensated by a robust control law. On the other hand, such faults are very hard or even impossible to detect and isolate.

For each faulty case, a set of 1000 Monte Carlo simulations has been carried out in order to assess the performance of the proposed FTC strategy. Thruster faults are uniformly distributed among all the 12 thrusters. In all cases, fault occurs at time $t_{f}=1000 \mathrm{~s}$ and is maintained. All the $(3 \times 1000)$ simulations were carried out under realistic conditions, i.e. the navigation unit is considered to deliver "non-perfect" state estimates. Therefore all signals used by the FDI scheme, NIPC algorithm and the $6 \mathrm{DoF}$ controller are replaced with their respective uncertain values. Time-varying delays induced by the CPDE device and spatial disturbances (e.g., solar radiation pressure, gravity gradient, and atmospheric drag assuming an exponential atmospheric model) are also considered.

For each run, the nominal model parameters were scattered within a specific limit (see Table 2 for details). The mass, the CoM and the inertia were scattered according to the normal distribution and truncated to the corresponding $3 \sigma$ values. The $1 \%$ multiplicative uncertainty on the thrusters forces models the uncertainty on the thruster rise times and the thruster misalignment phenomena. Because the real configuration matrix $\overline{\boldsymbol{B}}$ is never precisely known on-board, an uncertain configuration matrix is considered for on-board computational purposes (control law, FDI, CA). This matrix has been computed using a worst-case scenario when an offset of $-3 \mathrm{~cm}$ was added to each axis of the nominal CoM (see Table 2). A 10\% initial navigation uncertainty is considered on the Cartesian coordinates $\boldsymbol{x}_{p}$ (see Table 3).

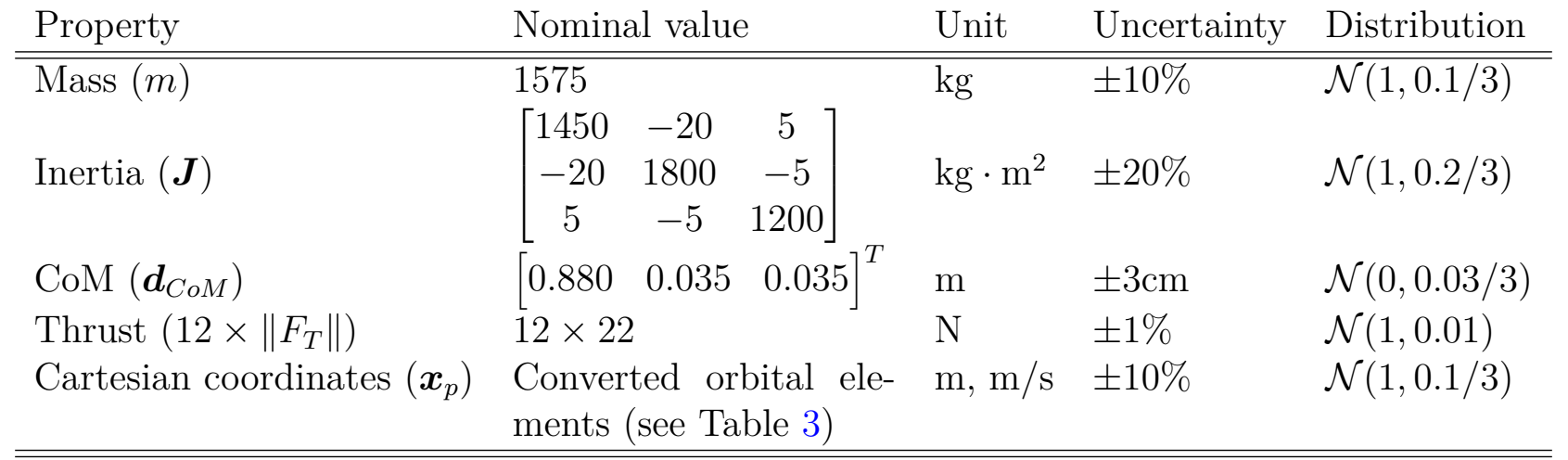

Table 2: Considered parameter uncertainties of the chaser spacecraft

To evaluate performance and reliability of the proposed FDI scheme, some statistical indices have been used like the mean detection delay and its corresponding deviation. The considered indices are listed below:

- $\mu\left(\tau_{d}\right) / \sigma\left(\tau_{d}\right)$ - mean/standard deviation (st.dev.) of the detection delay $\tau_{d}=t_{d}-t_{f}$,

- $\mu\left(\tau_{g}\right) / \sigma\left(\tau_{g}\right)-$ mean/st.dev. of the thruster group isolation delay $\tau_{g}=t_{g}-t_{d}$, 


\begin{tabular}{llll} 
Orbital parameter & Chaser & Target & Unit \\
\hline \hline Semimajor axis & 3893 & 3893 & $\mathrm{~km}$ \\
Eccentricity & 0 & 0 & $\mathrm{n} / \mathrm{a}$ \\
Inclination & 30 & 30 & $\mathrm{deg}$ \\
RAAN & 0 & 0 & $\mathrm{deg}$ \\
Argument of periapsis & 0 & 0 & $\mathrm{deg}$ \\
True anomaly & $-32.16 \times 10^{-5}$ & 0 & $\mathrm{deg}$ \\
\hline \hline
\end{tabular}

Table 3: Initial Keplerian orbital parameters of the chaser and target

- $\mu\left(\tau_{i}\right) / \sigma\left(\tau_{i}\right)$ - mean/st.dev. of the thruster isolation delay $\tau_{i}=t_{i}-t_{g}$,

- $\mu\left(\tau_{o}\right) / \sigma\left(\tau_{o}\right)$ - mean/st.dev. of the overall detection and isolation delay $\tau_{o}=t_{i}-t_{f}$,

- $p_{f}$ - FDI unit fail rate, i.e. the number of wrongly isolated thrusters divided by the total number of Monte Carlo runs (1000 for each fault scenario).

These performance indices are calculated for each fault case separately. Table 4 presents complete results obtained from the simulation campaign. This table demonstrates that the proposed FDI scheme is able to detect and isolate almost all considered thruster faults with good detection/isolation performances. In addition, it also shows a good reliability since no false detection/isolation has been revealed for the first two faulty scenarios $\left(p_{f}=0\right)$. Considering the thrust loss scenario, in about 110 simulation cases, the FDI unit failed to either detect or correctly isolate the faulty thruster. As it will be shown in the next, this fact does not violate any capture condition nor the mission success.

\begin{tabular}{lllllll} 
Metric & Fully open & & Leakage & & Thrust loss & \\
\hline \hline$\mu\left(\tau_{d}\right) / \sigma\left(\tau_{d}\right)$ & $2.36 / 0.14$ & $(\mathrm{~s})$ & $4.97 / 0.75$ & $(\mathrm{~s})$ & $48.44 / 53.29$ & $(\mathrm{~s})$ \\
$\mu\left(\tau_{g}\right) / \sigma\left(\tau_{g}\right)$ & $1.50 / 0.86$ & $(\mathrm{~s})$ & $1.75 / 0.37$ & $(\mathrm{~s})$ & $3.37 / 5.16$ & $(\mathrm{~s})$ \\
$\mu\left(\tau_{i}\right) / \sigma\left(\tau_{i}\right)$ & $0.40 / 0.00$ & $(\mathrm{~s})$ & $3.70 / 11.39$ & $(\mathrm{~s})$ & $4.20 / 8.21$ & $(\mathrm{~s})$ \\
$\mu\left(\tau_{o}\right) / \sigma\left(\tau_{o}\right)$ & $4.27 / 0.87$ & $(\mathrm{~s})$ & $10.41 / 11.71$ & $(\mathrm{~s})$ & $56.01 / 54.57$ & $(\mathrm{~s})$ \\
$p_{f}$ & 0 & & 0 & & 0.11 & \\
\hline \hline
\end{tabular}

Table 4: FDI performances based on $3 \times 1000$ Monte Carlo runs

Figures 5a-9b illustrate the fault tolerant capabilities of the proposed technique. The capture conditions in terms of position and velocities are given in Fig. 5a, Fig. 7a, and Fig. 9a for fully open thruster, leaking thruster and efficiency loss thruster fault, respectively. Figure 5b, Fig. 7b and Fig. 9b illustrate that in all faulty cases the chaser maintains the nominal trajectory (i.e. stays inside the rendezvous corridor) and that the chaser keeps its attitude pointing towards the target, thus, leading to a successful capture. Finally, Fig. 6b, Fig. 8b and Fig. 10b show that the proposed strategy is able to meet the required $3 \sigma$ capture accuracy in terms of angular misalignment and angular rate errors. 


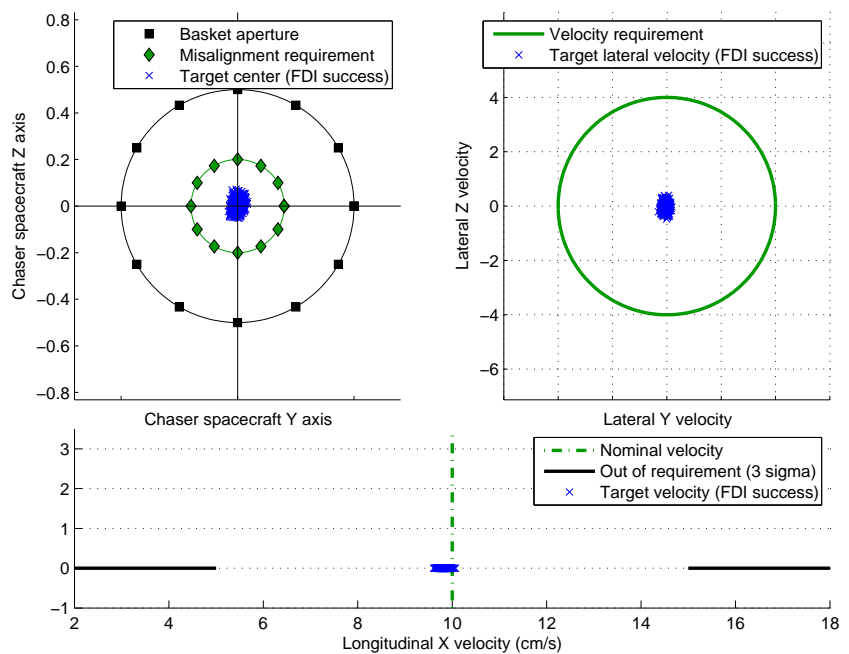

(a) Position misalignment (top left), lateral velocity (top right) and longitudinal velocity (bottom)

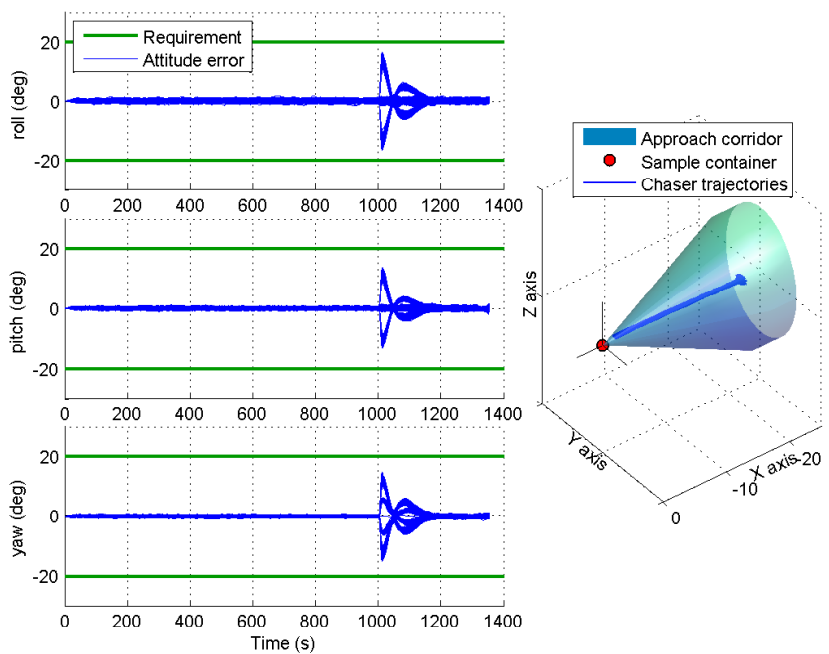

(b) Chaser's attitude error (left) and trajectory inside the rendezvous corridor (right)

Figure 5: Capture position requirements and GNC performances for fault case 1

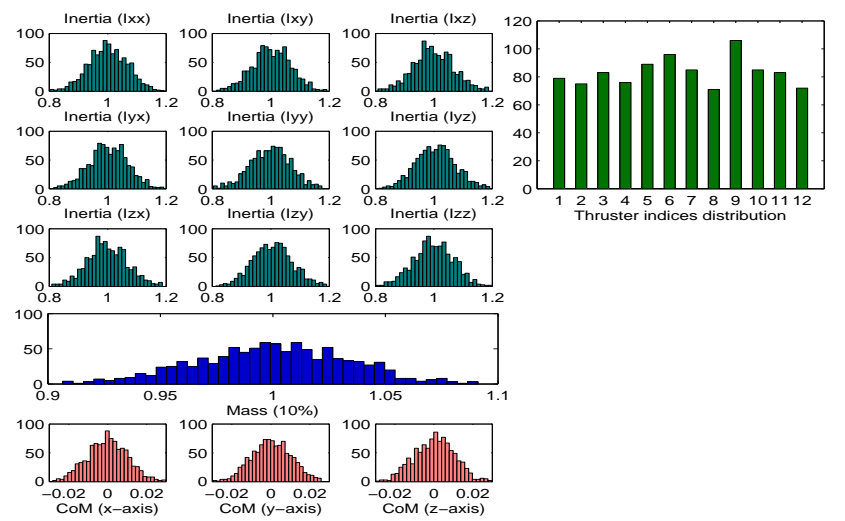

(a) Inertia (top left), mass (middle left), CoM (bottom left) and thruster indices (top right) distribution
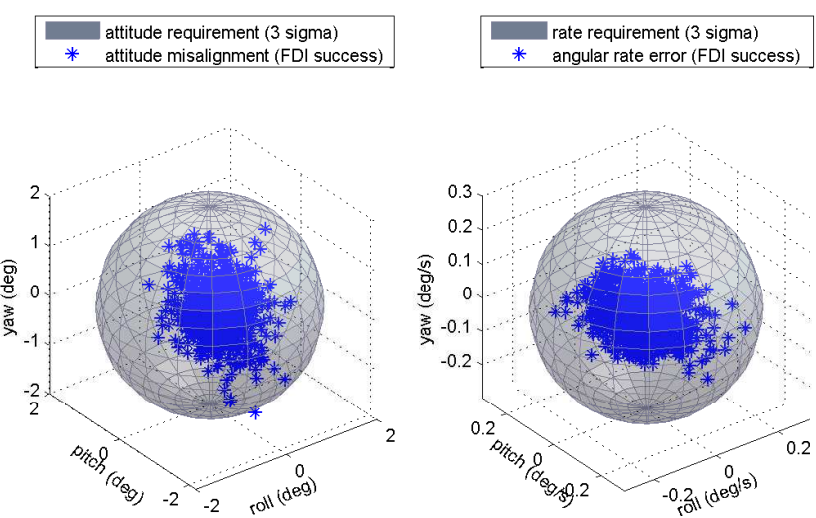

(b) Angular misalignment (left) and angular rate erro (right) at capture

Figure 6: Considered distributions and capture angular requirements for fault case 1 


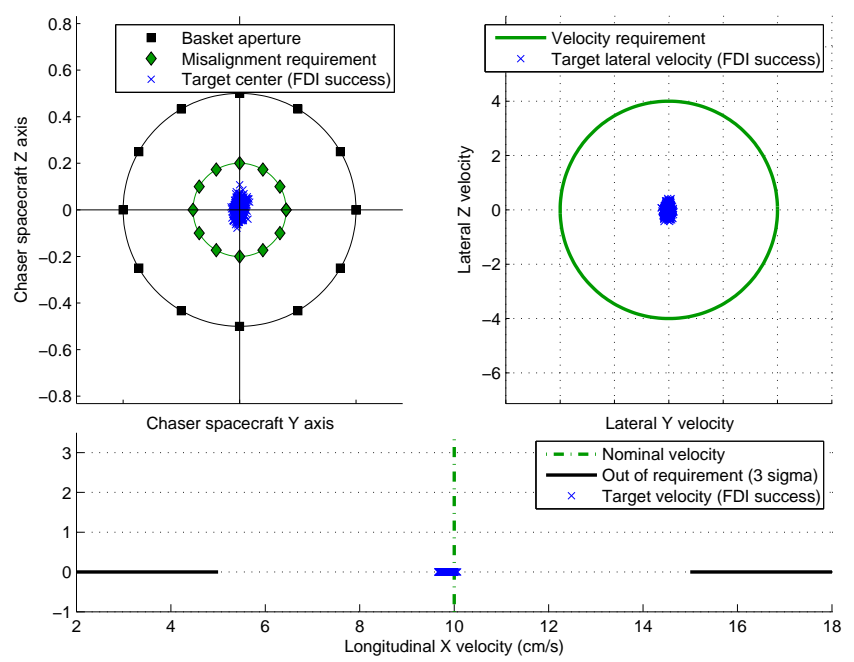

(a) Position misalignment (top left), lateral velocity (top right) and longitudinal velocity (bottom)

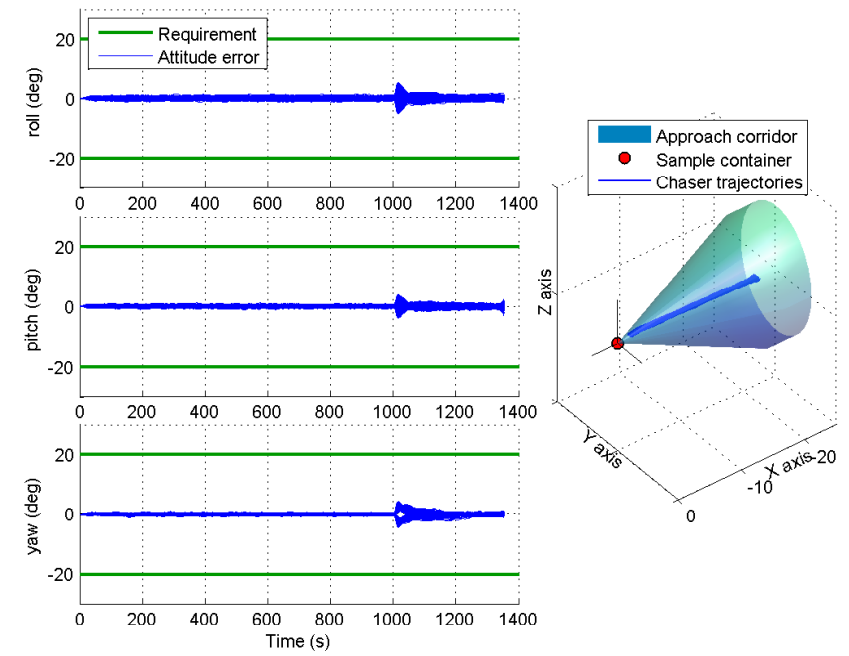

(b) Chaser's attitude error (left) and trajectory inside the rendezvous corridor (right)

Figure 7: Capture position requirements and GNC performances for fault case 2

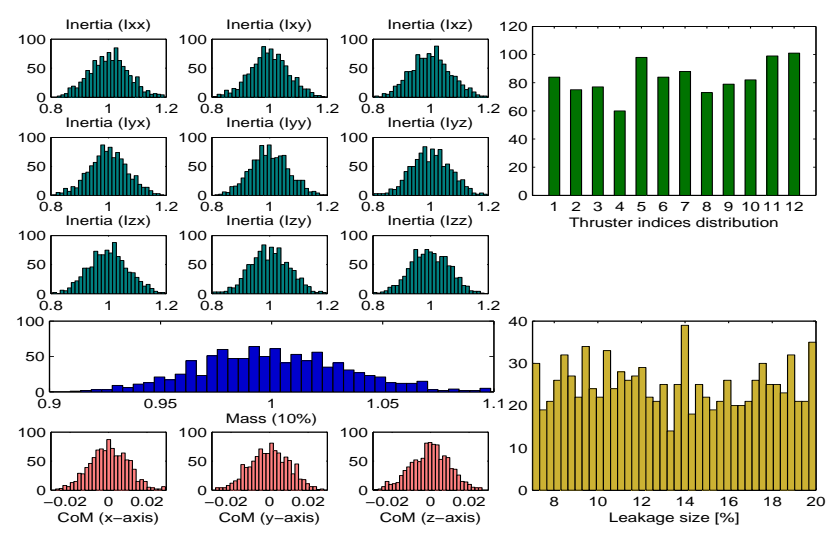

(a) Inertia (top left), mass (middle left), CoM (bottom left), thruster indices (top right) and leakage size (bottom right) distribution
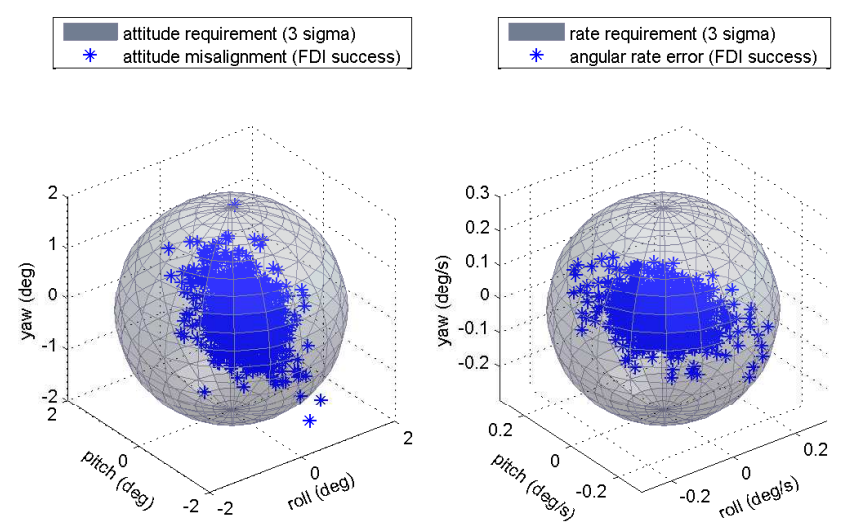

(b) Angular misalignment (left) and angular rate error (right) at capture

Figure 8: Considered distributions and capture angular requirements for fault case 2 


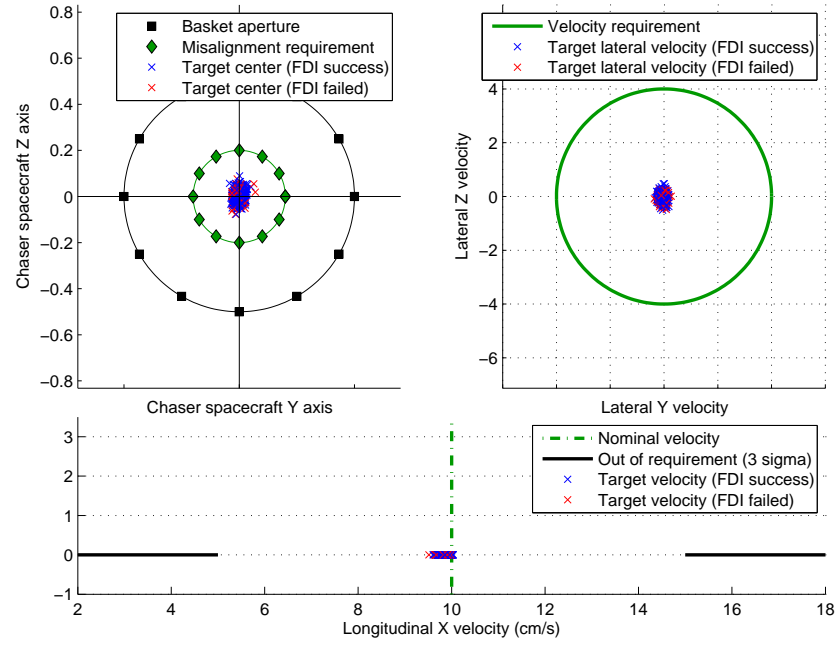

(a) Position misalignment (top left), lateral velocity (top right) and longitudinal velocity (bottom)

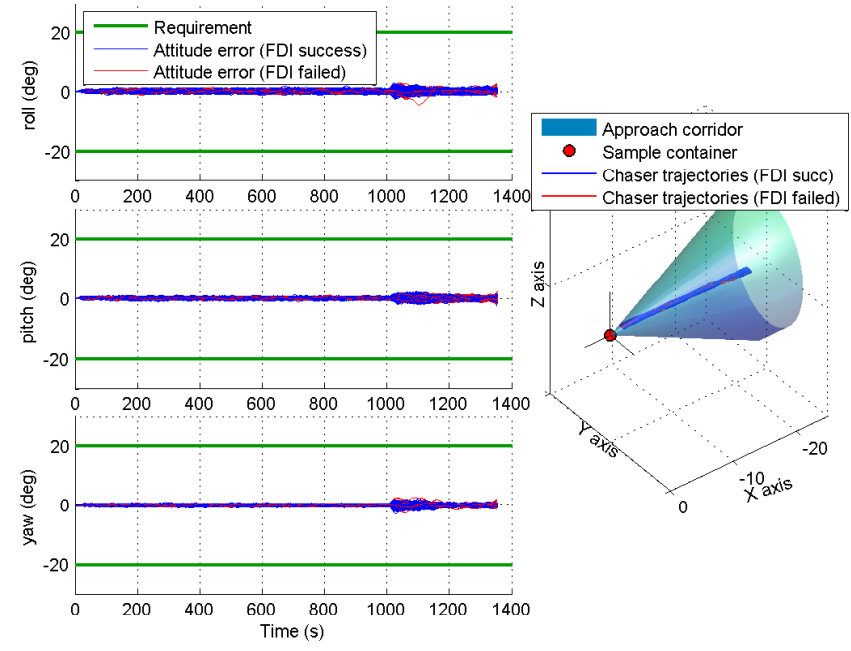

(b) Chaser's attitude error (left) and trajectory inside the rendezvous corridor (right)

Figure 9: Capture position requirements and GNC performances for fault case 3

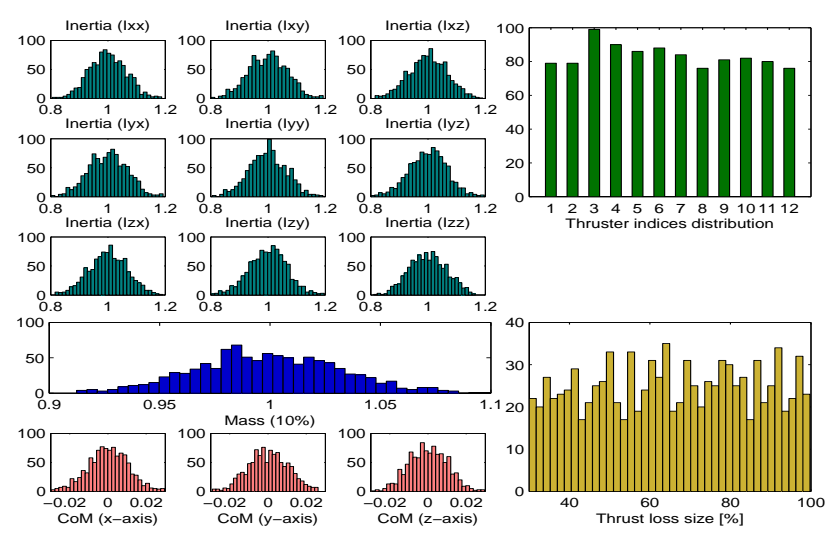

(a) Inertia (top left), mass (middle left), CoM (bottom left), thruster indices (top right) and thrust loss size (bottom right) distribution
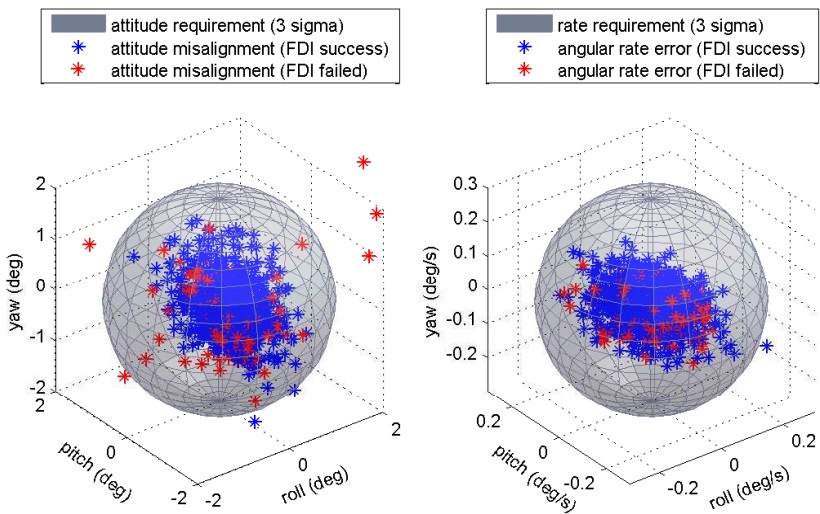

(b) Angular misalignment (left) and angular rate error (right) at capture

Figure 10: Considered distributions and capture angular requirements for fault case 3 
Note that the early detection of the occurrence of incipient or small size thruster faults (e.g., small propellant leakage or small thrust loss) is clearly more difficult. Another problem can arise when a fully blocked thruster (i.e. $m_{l o s s}=1$ ) is not commanded and thus a fault detection is almost impossible. As seen in Fig. 9a and Fig. 9b, despite the fact that in some cases the FDI unit failed, the required capture tolerances and attitude/trajectory conditions are fully met.

On the other hand, in some particular cases, the attitude misalignment requirement (3 sigma) is not met even if the FDI unit succeeded. This can be the case when it takes too long for the FDI unit to detect and/or isolate the faulty thruster or when the control accuracy is very degraded, e.g., due to a worst case uncertainty or strong disturbance. In such cases, the solution consists in a corrective maneuver (e.g. triggering a collision avoidance maneuver) that is engaged at the higher level of the fault management unit, see (LePeuvédic et al., 2014).

\section{Conclusion}

In this paper, a systematic procedure has been presented for the theoretical design and application of a model-based approach to FDI/CA-based FTC of an autonomous rendezvous system in the terminal phase. The aim was to detect and isolate a single thruster fault affecting the chaser propulsion system and to accommodate it as quick as possible. The proposed FDI scheme consists of a robust fault detector and a NUIO and EKF-based hierarchical isolation logic. The NUIO gains are given by solving an LMI optimization problem, which ensures maximization of the admissible Lipschitz constant while simultaneously satisfying an $\mathcal{L}_{2}$ gain bound and pole constraints on observer dynamics. The $\mathcal{L}_{2}$ attenuation is considered to minimize the effect of the uncertain inertia on the state estimation error. The NUIO design together with the derivation of the uncertain inertia inverse factorization can be considered as a contribution to the theory. The thruster fault tolerance is achieved by an improved version of the the NIPC control allocation algorithm scheduled by the robust FDI scheme. A Monte Carlo simulation campaign has been performed to assess the performance and robustness of the FDI/CA-based FTC system subject to parameter uncertainties, spatial disturbances, delays and imperfect navigation. The obtained results indicate that for all the considered fault profiles, which are those considered to be the most relevant by the industrial partners, the proposed strategy can carry out the terminal rendezvous successfully and meet all the required capture specifications.

\section{Acknowledgement}

The authors would like to thank the ESA (Guidance, Navigation and Control Section at European Space Research and Technology Centre) and Thales Alenia Space France (Research and Technology/Science and Observation within the Research and Development Department) for providing the funding that made this research possible, through the ESA Networking/Partnering Initiative (NPI) Program. 


\section{Appendix A. Proof of Proposition 1}

To prove Proposition 1, the following lemma is introduced first:

Lemma 1 (Neumann series of a matrix, Chatelin (1983)). Consider a square matrix $\boldsymbol{A}$ such that $\|\boldsymbol{A}\|<1$. Let $\lambda$ be any eigenvalue of $\boldsymbol{A}$. It is clear that $(\boldsymbol{I}-\boldsymbol{A})$ is invertible if $\lambda \neq 1, \forall \lambda \in \Lambda(\boldsymbol{A})$. The condition $\|\boldsymbol{A}\|<1$ implies that $|\lambda|<1, \forall \lambda \in \Lambda(\boldsymbol{A})$. Thus, $(\boldsymbol{I}-\boldsymbol{A})$ is invertible and the Neumann series

$$
(\boldsymbol{I}-\boldsymbol{A})^{-1}=\sum_{k=0}^{\infty} \boldsymbol{A}^{k}=\boldsymbol{I}+\boldsymbol{A}+\boldsymbol{A}^{2}+\ldots
$$

converges. When $\|\boldsymbol{A}\| \geq 1,(\boldsymbol{I}-\boldsymbol{A})$ is still invertible if $\lambda \neq 1, \forall \lambda \in \Lambda(\boldsymbol{A})$, but the Neumann series does not converge because $\lim _{k \rightarrow \infty} \boldsymbol{A}^{k} \neq \mathbf{0}$.

Proof of Lemma 1 Since $\|\boldsymbol{A}\|<1$, the series $\sum_{k=0}^{\infty}\|\boldsymbol{A}\|^{k}$ converges. Since $\left\|\boldsymbol{A}^{h}\right\| \leq\|\boldsymbol{A}\|^{h}$, the series $\sum_{k=0}^{\infty} \boldsymbol{A}^{k}$ converges, too. Denote by $\boldsymbol{Z}$ its limit. $\boldsymbol{Z} \boldsymbol{A}=\boldsymbol{A} \boldsymbol{Z}=\sum_{k=0}^{\infty} \boldsymbol{A}^{k+1}$; therefore $(\boldsymbol{I}-\boldsymbol{A}) \boldsymbol{Z}=\boldsymbol{Z}(\boldsymbol{I}-\boldsymbol{A})=\boldsymbol{I}$, which proves (A.1).

The real inertia matrix $\boldsymbol{J}$ is always invertible and symmetric, thus $\boldsymbol{J}_{0}$ and $\boldsymbol{J}_{0}+\boldsymbol{R}_{J}^{*} \boldsymbol{\Delta}_{J}^{*} \boldsymbol{S}_{J}$ are invertible and symmetric too. Now, multiplying (20) by $\boldsymbol{J}_{0}^{-1}$ from the left yields

$$
\boldsymbol{J}_{0}^{-1} \boldsymbol{J}=\boldsymbol{I}+\boldsymbol{J}_{0}^{-1} \boldsymbol{R}_{J}^{*} \boldsymbol{\Delta}_{J}^{*} \boldsymbol{S}_{J}
$$

inverting both sides gives

$$
\boldsymbol{J}^{-1} \boldsymbol{J}_{0}=\left(\boldsymbol{I}+\boldsymbol{J}_{0}^{-1} \boldsymbol{R}_{J}^{*} \boldsymbol{\Delta}_{J}^{*} \boldsymbol{S}_{J}\right)^{-1}
$$

Since $\boldsymbol{\Delta}_{J}^{* T} \boldsymbol{\Delta}_{J}^{*} \leq \boldsymbol{I} \Rightarrow\left\|\boldsymbol{\Delta}_{J}^{*}\right\| \leq 1$, the following bound yields

$$
\left\|\boldsymbol{J}_{0}^{-1} \boldsymbol{R}_{J}^{*} \boldsymbol{\Delta}_{J}^{*} \boldsymbol{S}_{J}\right\| \leq\left\|\boldsymbol{J}_{0}^{-1} \boldsymbol{R}_{J}^{*}\right\|\left\|\boldsymbol{\Delta}_{J}^{*}\right\|\left\|\boldsymbol{S}_{J}\right\| \leq\left\|\boldsymbol{J}_{0}^{-1} \boldsymbol{R}_{J}^{*}\right\|\left\|\boldsymbol{S}_{J}\right\|
$$

Thus, if $\left\|\boldsymbol{J}_{0}^{-1} \boldsymbol{R}_{J}^{*}\right\|\left\|\boldsymbol{S}_{J}\right\|<1$, then the right-hand side of (A.3) can be expressed according to to Lemma 1 as follows

$$
\left(\boldsymbol{I}-\left(-\boldsymbol{J}_{0}^{-1} \boldsymbol{R}_{J}^{*} \boldsymbol{\Delta}_{J}^{*} \boldsymbol{S}_{J}\right)\right)^{-1}=\sum_{k=0}^{\infty}(-1)^{k}\left(\boldsymbol{J}_{0}^{-1} \boldsymbol{R}_{J}^{*} \boldsymbol{\Delta}_{J}^{*} \boldsymbol{S}_{J}\right)^{k}
$$

Pre-multiplying (A.3) by $\boldsymbol{J}_{0}^{-1}$ from the right and substituting (A.5) gives

$$
\begin{gathered}
\boldsymbol{J}^{-1}=\sum_{k=0}^{\infty}(-1)^{k}\left(\boldsymbol{J}_{0}^{-1} \boldsymbol{R}_{J}^{*} \boldsymbol{\Delta}_{J}^{*} \boldsymbol{S}_{J}\right)^{k} \boldsymbol{J}_{0}^{-1} \\
=\boldsymbol{J}_{0}^{-1}+\sum_{k=1}^{\infty}(-1)^{k}\left(\boldsymbol{J}_{0}^{-1} \boldsymbol{R}_{J}^{*} \boldsymbol{\Delta}_{J}^{*} \boldsymbol{S}_{J}\right)^{k} \boldsymbol{J}_{0}^{-1}=\boldsymbol{J}_{0}^{-1}+\boldsymbol{R}_{1} \boldsymbol{\Delta}_{1} \boldsymbol{S}_{1}
\end{gathered}
$$

where

$$
\begin{aligned}
\boldsymbol{R}_{1} & =\boldsymbol{J}_{0}^{-1} \boldsymbol{R}_{J}^{*} \\
\boldsymbol{S}_{1} & =\boldsymbol{S}_{J} \boldsymbol{J}_{0}^{-1} \\
\boldsymbol{\Delta}_{1} & =\boldsymbol{\Delta}_{J}^{*}\left(-\boldsymbol{I}+\boldsymbol{S}_{J} \boldsymbol{J}_{0}^{-1} \boldsymbol{R}_{J}^{*} \boldsymbol{\Delta}_{J}^{*}-\left(\boldsymbol{S}_{J} \boldsymbol{J}_{0}^{-1} \boldsymbol{R}_{J}^{*} \boldsymbol{\Delta}_{J}^{*}\right)^{2}+\ldots\right)
\end{aligned}
$$


It is needed to check if $\boldsymbol{\Delta}_{1}^{T} \boldsymbol{\Delta}_{1} \leq 1$. Considering the worst-case uncertainty, i.e. $\boldsymbol{\Delta}_{J}^{*}=\boldsymbol{I}$, and inserting it in (A.9) yields to

$$
\overline{\boldsymbol{\Delta}}_{1}=-\boldsymbol{I}+\boldsymbol{S}_{J} \boldsymbol{J}_{0}^{-1} \boldsymbol{R}_{J}^{*}-\left(\boldsymbol{S}_{J} \boldsymbol{J}_{0}^{-1} \boldsymbol{R}_{J}^{*}\right)^{2}+\ldots=-\sum_{k=0}^{\infty}(-1)^{k}\left(\boldsymbol{S}_{J} \boldsymbol{J}_{0}^{-1} \boldsymbol{R}_{J}^{*}\right)^{k}
$$

which gives the upper bound of $\boldsymbol{\Delta}_{1}$, i.e. $\left\|\boldsymbol{\Delta}_{1}\right\| \leq\left\|\bar{\Delta}_{1}\right\|$. According to Lemma 1 , the right-hand side of (A.10) is equivalent to

$$
\overline{\boldsymbol{\Delta}}_{1}=-\sum_{k=0}^{\infty}(-1)^{k}\left(\boldsymbol{S}_{J} \boldsymbol{J}_{0}^{-1} \boldsymbol{R}_{J}^{*}\right)^{k}=-\left(\boldsymbol{I}+\boldsymbol{S}_{J} \boldsymbol{J}_{0}^{-1} \boldsymbol{R}_{J}^{*}\right)^{-1}
$$

if $\left\|\boldsymbol{S}_{J} \boldsymbol{J}_{0}^{-1} \boldsymbol{R}_{J}^{*}\right\|<1$, which is true since $\left\|\boldsymbol{S}_{J} \boldsymbol{J}_{0}^{-1} \boldsymbol{R}_{J}^{*}\right\| \leq\left\|\boldsymbol{J}_{0}^{-1} \boldsymbol{R}_{J}^{*}\right\|\left\|\boldsymbol{S}_{J}\right\|<1$. It is obvious that $\left\|\overline{\boldsymbol{\Delta}}_{1}\right\|=\left\|\left(\boldsymbol{I}+\boldsymbol{S}_{J} \boldsymbol{J}_{0}^{-1} \boldsymbol{R}_{J}^{*}\right)^{-1}\right\|>1$, thus a new scaling matrix $\boldsymbol{W}_{2}$ must be introduced such that

$$
\boldsymbol{\Delta}_{1}=\boldsymbol{W}_{2} \boldsymbol{\Delta}_{2}, \quad \boldsymbol{\Delta}_{2}^{T} \boldsymbol{\Delta}_{2} \leq \boldsymbol{I}
$$

where $\boldsymbol{\Delta}_{2}$ is unknown. One of the possible choice of $\boldsymbol{W}_{2}$ is to take the norm upper bound of $\Delta_{1}$, i.e.

$$
\boldsymbol{W}_{2}=\left\|\bar{\Delta}_{1}\right\| \boldsymbol{I}=\left\|\left(\boldsymbol{I}+\boldsymbol{S}_{J} \boldsymbol{J}_{0}^{-1} \boldsymbol{R}_{J}^{*}\right)^{-1}\right\| \boldsymbol{I}
$$

Then, the following holds

$$
\left\|\boldsymbol{\Delta}_{1}\right\|=\left\|\boldsymbol{W}_{2} \boldsymbol{\Delta}_{2}\right\|=\left\|\bar{\Delta}_{1}\right\|\left\|\boldsymbol{\Delta}_{2}\right\| \leq\left\|\overline{\boldsymbol{\Delta}}_{1}\right\| \Rightarrow \boldsymbol{\Delta}_{2}^{T} \boldsymbol{\Delta}_{2} \leq \boldsymbol{I}
$$

Inserting (A.12) into (A.6) and setting $\boldsymbol{R}_{2}=\boldsymbol{R}_{1} \boldsymbol{W}_{2}, \boldsymbol{S}_{2}=\boldsymbol{S}_{1}$, (A.6) yields (21).

\section{Appendix B. Proof of Theorem 1}

In the proof of Theorem 1, the following lemma is used:

Lemma 2 (Zhou and Khargonekar (1988)). Let $\boldsymbol{D}, \boldsymbol{F}$, and $\boldsymbol{\Sigma}(t)$ being matrices with appropriate dimensions. If $\boldsymbol{\Sigma}^{T}(t) \boldsymbol{\Sigma}(t) \leq \boldsymbol{I}$, then for any scalar $\epsilon>0$ the following inequality holds:

$$
\boldsymbol{D} \boldsymbol{\Sigma}(t) \boldsymbol{F}+\boldsymbol{F}^{T} \boldsymbol{\Sigma}^{T}(t) \boldsymbol{D}^{T} \leq \epsilon^{-1} \boldsymbol{D} \boldsymbol{D}^{T}+\epsilon \boldsymbol{F}^{T} \boldsymbol{F}
$$

Proof of Lemma 2 It can be verified that the following yields

$$
\left(\epsilon^{\frac{1}{2}} \boldsymbol{D}^{T}-\epsilon^{\frac{1}{2}} \boldsymbol{\Sigma}(t) \boldsymbol{F}\right)^{T}\left(\epsilon^{\frac{1}{2}} \boldsymbol{D}^{T}-\epsilon^{\frac{1}{2}} \boldsymbol{\Sigma}(t) \boldsymbol{F}\right) \geq 0
$$

then expanding the above yields

$$
\epsilon^{-1} \boldsymbol{F}^{T} \boldsymbol{\Sigma}^{T}(t) \boldsymbol{\Sigma}(t) \boldsymbol{F}+\epsilon \boldsymbol{D} \boldsymbol{D}^{T} \geq \boldsymbol{D} \boldsymbol{\Sigma}(t) \boldsymbol{F}+\boldsymbol{F}^{T} \boldsymbol{\Sigma}^{T}(t) \boldsymbol{D}^{T}
$$

It is obvious that $\|\boldsymbol{\Sigma}\| \leq 1 \Leftrightarrow \lambda_{\max }\left(\boldsymbol{\Sigma}^{T} \boldsymbol{\Sigma}\right) \leq 1 \Leftrightarrow \boldsymbol{\Sigma}^{T} \boldsymbol{\Sigma} \leq \boldsymbol{I}$, thus

$$
\epsilon \boldsymbol{D}^{T}+\epsilon^{-1} \boldsymbol{E}^{T} \boldsymbol{E} \geq \epsilon^{-1} \boldsymbol{F}^{T} \boldsymbol{\Sigma}^{T}(t) \boldsymbol{\Sigma}(t) \boldsymbol{F}+\epsilon \boldsymbol{D} \boldsymbol{D}^{T} \geq \boldsymbol{D} \boldsymbol{\Sigma}(t) \boldsymbol{F}+\boldsymbol{F}^{T} \boldsymbol{\Sigma}^{T}(t) \boldsymbol{D}^{T}
$$


To proceed with the proof of Theorem 1, assume that $\boldsymbol{H}$ is chosen such that (33) holds. Under the assumption that $\boldsymbol{\Delta} \boldsymbol{B}=\boldsymbol{R}_{2} \boldsymbol{\Delta}_{2} \boldsymbol{S}_{2} \boldsymbol{B}_{T}$ with $\boldsymbol{\Delta}_{2}^{T} \boldsymbol{\Delta}_{2} \leq \boldsymbol{I}$, the error dynamics of the NUIO can be rewritten as

$$
\dot{e}=\boldsymbol{N e}+\boldsymbol{M}(\boldsymbol{\Phi}-\hat{\boldsymbol{\Phi}})+\boldsymbol{M R _ { 2 }} \Delta_{2} \boldsymbol{S}_{2} \boldsymbol{B}_{T} \boldsymbol{u}
$$

where $\boldsymbol{\Phi}$ and $\hat{\boldsymbol{\Phi}}$ stand for $\boldsymbol{\Phi}(\boldsymbol{x})$ and $\boldsymbol{\Phi}(\hat{\boldsymbol{x}})$, respectively. Considering the quadratic Lyapunov function $V(t)=\boldsymbol{e}(t)^{T} \boldsymbol{P} \boldsymbol{e}(t)$, the time derivative of $V(t)$ along the trajectory of (B.2) is given by

$$
\dot{V}=\boldsymbol{e}^{T}\left(\boldsymbol{N}^{T} \boldsymbol{P}+\boldsymbol{P} \boldsymbol{N}\right) \boldsymbol{e}+2 \boldsymbol{e}^{T} \boldsymbol{P} \boldsymbol{M}(\boldsymbol{\Phi}-\hat{\boldsymbol{\Phi}})+2 \boldsymbol{e}^{T} \boldsymbol{P} \boldsymbol{M} \boldsymbol{R}_{2} \boldsymbol{\Delta}_{2} \boldsymbol{S}_{2} \boldsymbol{B}_{T} \boldsymbol{u}
$$

Using the Lipschitz condition stated in Assumption 1 and Lemma 2 with $\epsilon=1$ it follows that

$$
\begin{aligned}
& 2 \boldsymbol{e}^{T} \boldsymbol{P} \boldsymbol{M}(\hat{\boldsymbol{\Phi}}-\boldsymbol{\Phi}) \leq 2 \gamma\left\|\boldsymbol{e}^{T} \boldsymbol{P} \boldsymbol{M}\right\|\|\boldsymbol{e}\| \leq \boldsymbol{e}^{T} \boldsymbol{P} \boldsymbol{M} \boldsymbol{M}^{T} \boldsymbol{P} \boldsymbol{e}+\gamma^{2} \boldsymbol{e}^{T} \boldsymbol{e} \\
& 2 \boldsymbol{e}^{T} \boldsymbol{P} \boldsymbol{M} \boldsymbol{R}_{2} \boldsymbol{\Delta}_{2} \boldsymbol{S}_{2} \boldsymbol{B}_{T} \boldsymbol{u} \leq \boldsymbol{e}^{T} \boldsymbol{P} \boldsymbol{M} \boldsymbol{R}_{2} \boldsymbol{R}_{2}^{T} \boldsymbol{M}^{T} \boldsymbol{P} \boldsymbol{e}+\boldsymbol{u}^{T}\left(\boldsymbol{S}_{2} \boldsymbol{B}_{T}\right)^{T} \boldsymbol{S}_{2} \boldsymbol{B}_{T} \boldsymbol{u}
\end{aligned}
$$

and (B.3) can be bounded as follows

$$
\dot{\boldsymbol{V}} \leq \boldsymbol{e}^{T}\left(\boldsymbol{N}^{T} \boldsymbol{P}+\boldsymbol{P} \boldsymbol{N}+\boldsymbol{P} \boldsymbol{M}\left(\boldsymbol{I}+\boldsymbol{R}_{2} \boldsymbol{R}_{2}^{T}\right) \boldsymbol{M}^{T} \boldsymbol{P}+\gamma^{2} \boldsymbol{I}\right) \boldsymbol{e}+\boldsymbol{u}^{T}\left(\boldsymbol{S}_{2} \boldsymbol{B}_{T}\right)^{T} \boldsymbol{S}_{2} \boldsymbol{B}_{T} \boldsymbol{u}
$$

Let's consider the $\mathcal{H}_{\infty}$ performance criteria

$$
\min _{\kappa}: \int_{0}^{T} \boldsymbol{e}^{T}(t) \boldsymbol{e}(t) d t \leq \kappa^{2} \int_{0}^{T} \boldsymbol{u}^{T}(t) \boldsymbol{u}(t) d t \quad \forall T \geq 0
$$

then it is straightforward to verify that the $\mathcal{L}_{2}$ gain from $\Delta \boldsymbol{B} \boldsymbol{u}$ to $\boldsymbol{e}$ is bounded by $\kappa>0$ if and only if

$$
\left[\begin{array}{cc}
\boldsymbol{\Psi}_{1} & \mathbf{0} \\
* & \boldsymbol{\Psi}_{2}
\end{array}\right]<0
$$

with

$$
\begin{aligned}
& \boldsymbol{\Psi}_{1}=\boldsymbol{N}^{T} \boldsymbol{P}+\boldsymbol{P} \boldsymbol{N}+\left(1+\gamma^{2}\right) \boldsymbol{I}+\boldsymbol{P} \boldsymbol{M}\left(\boldsymbol{I}+\boldsymbol{R}_{2} \boldsymbol{R}_{2}^{T}\right) \boldsymbol{M}^{T} \boldsymbol{P} \\
& \boldsymbol{\Psi}_{2}=\left(\boldsymbol{S}_{2} \boldsymbol{B}_{T}\right)^{T} \boldsymbol{S}_{2} \boldsymbol{B}_{T}-\kappa^{2} \boldsymbol{I}
\end{aligned}
$$

Then, by virtue of the Schur's complement lemma, (B.6) is equivalent to

$$
\left[\begin{array}{ccccc}
\boldsymbol{N}^{T} \boldsymbol{P}+\boldsymbol{P} \boldsymbol{N}+\left(1+\gamma^{2}\right) \boldsymbol{I} & \boldsymbol{P} \boldsymbol{M} & \boldsymbol{P} \boldsymbol{M} \boldsymbol{R}_{2} & \mathbf{0} & \mathbf{0} \\
* & -\boldsymbol{I} & \mathbf{0} & \mathbf{0} & \mathbf{0} \\
* & * & -\boldsymbol{I} & \mathbf{0} & \mathbf{0} \\
* & * & * & -\kappa^{2} \boldsymbol{I} & \boldsymbol{S}_{2} \boldsymbol{B}_{T} \\
* & * & * & * & -\boldsymbol{I}
\end{array}\right]<0
$$

It can be seen that there is no systematic way to obtain the observer parameters directly from (B.7) due to coupled terms. To reformulate (B.7) as an LMI, $\boldsymbol{H}$ is substituted by (34), and use the following assignments $\overline{\boldsymbol{Y}}=\boldsymbol{P} \boldsymbol{Y}, \overline{\boldsymbol{K}}=\boldsymbol{P} \boldsymbol{K}$ and $\xi=\gamma^{2}$. Additionally, it is desired to achieve the maximum possible Lipschitz constant $\gamma^{*}$ and simultaneously to respect the constraint $\gamma^{*} \geq \gamma$. This constraint can be rewritten by defining a new variable $\xi=\left(\gamma^{*}\right)^{2}$ as $\xi-\gamma^{2} \geq 0$. Then, using the Schur's complement, (38) follows. It is then obvious that maximizing $\xi$ is equivalent to maximizing $\gamma^{*}$. This concludes the proof of Theorem 1 . 


\section{References}

Abbaszadeh, M., Marquez, H.J., 2009. LMI optimization approach to robust $H_{\infty}$ observer design and static output feedback stabilization for discrete-time nonlinear uncertain systems. International Journal of Robust and Nonlinear Control 19, 313-340. doi:10.1002/rnc.1310.

Alwi, H., Edwards, C., 2008. Fault tolerant control using sliding modes with on-line control allocation. Automatica 44,1859-1866. doi:10.1016/j.automatica.2007.10.034.

Alwi, H., Edwards, C., Marcos, A., 2010. FDI for a mars orbiting satellite based on a sliding mode observer scheme, in: Conference on Control and Fault-Tolerant Systems (SysTol), IEEE, Nice, France. pp. 125-130. doi:10.1109/SYSTOL.2010.5676035.

Bajpai, G., Chang, B., Lau, A., 2001. Reconfiguration of flight control systems for actuator failures. IEEE Aerospace and Electronic Systems Magazine 16, 29-33. doi:10.1109/62. 949534.

Basseville, M., Nikiforov, I., 1993. Detection of Abrupt Changes: Theory and Application. Prentice Hall, Englewood Cliffs, NJ.

Beaty, D., Grady, M., May, L., Gardini, B., 2008. Preliminary planning for an international Mars Sample Return mission. Technical Report. Report of the International Mars Architecture for the Return of Samples (iMARS) Working Group.

Blanke, M., Kinnaert, M., Lunze, J., Staroswiecki, M., 2006. Diagnosis and Fault-Tolerant Control. Springer Verlag, Berlin.

Boada, J., Prieur, C., Tarbouriech, S., Pittet, C., Charbonnel, C., 2010. Multi-saturation antiwindup structure for satellite control, in: Proc. American Control Conference, Baltimore, USA. pp. 5979-5984. doi:10.1109/ACC. 2010.5531254.

Bodson, M., 2002. Evaluation of optimization methods for control allocation. Journal of Guidance, Control and Dynamics 25, 703-711. doi:10.2514/2.4937.

Bodson, M., Groszkiewicz, J., 1997. Multivariable adaptive control algorithms for reconfigurable flight control. IEEE Transactions on Control Systems Technology 5, 217-229. doi:10.1109/ 87.556026.

Caglayan, A., Allen, S., Wehmuller, K., 1988. Evaluation of a second generation reconfiguration strategy for aircraft flight control systems subjected to actuator failure/surface damage, in: IEEE National Aerospace and Electronics Conference, pp. 520-590. doi:10.1109/NAECON . 1988.195057.

Camacho, E., Bordons, C., 1999. Model Predictive Control. Springer, London. 
Chatelin, F., 1983. Spectral Approximation of Linear Operators. Academic Press, Society for Industrial and Applied Mathematics, New York.

Chen, J., Patton, R., 1999. Robust model-based fault diagnosis for dynamic systems. Kluwer Academic Publishers, Dordrecht. doi:10.1007/978-1-4615-5149-2.

Chen, W., Saif, M., 2007. Observer-based fault diagnosis of satellite systems subject to timevarying thruster faults. Journal of Dynamic Systems, Measurement and Control 129, 352-356. doi:10.1115/1.2719773.

Chilali, M., Gahinet, P., 1996. $H_{\infty}$ design with pole placement constraints: An LMI approach. IEEE Transactions on Automatic Control 41, 358-367. doi:10.1109/9.486637.

Ding, S.X., 2013. Model-based Fault Diagnosis Techniques: Design Schemes, Algorithms, and Tools. 2nd ed., Springer-Verlag, London. doi:10.1007/978-1-4471-4799-2.

Efimov, D., Cieslak, J., Henry, D., 2013. Supervisory fault tolerant control with mutual performance optimization. International Journal of Adaptive Control and Signal Processing 27, 251-279. doi:10.1002/acs . 2296.

Falcoz, A., Boquet, F., Dinh, M., Polle, B., Flandin, G., Bornschlegl, E., 2010a. Robust fault diagnosis for spacecraft: Application to LISA pathfinder experiment, in: 18th IFAC Symposium on Automatic Control in Aerospace, IFAC, Nara, Japan. pp. 404-409. doi:10. 3182/20100906-5-JP-2022.00069.

Falcoz, A., Boquet, F., Flandin, G., 2010b. Robust $H_{\infty} / H_{-}$thruster failure detection and isolation with application to the lisa pathfinder spacecraft, in: AIAA Guidance, Navigation, and Control Conference, AIAA, Toronto, Ontario. doi:10.2514/6.2010-7906.

Fonod, R., Henry, D., Bornschlegl, E., Charbonnel, C., 2013. Robust fault detection for systems with electronic induced delays: Application to the rendezvous phase of the MSR mission, in: 12th European Control Conference, Zürich, Switzerland. pp. 1439-1444.

Fonod, R., Henry, D., Bornschlegl, E., Charbonnel, C., 2014a. Thruster fault detection, isolation and accommodation for an autonomous spacecraft, in: 19th IFAC World Congress, Cape Town, South Africa. pp. 10543-10548. doi:10.3182/20140824-6-ZA-1003.02144.

Fonod, R., Henry, D., Charbonnel, C., Bornschlegl, E., 2014b. A class of nonlinear unknown input observer for fault diagnosis: Application to fault tolerant control of an autonomous spacecraft, in: 10th UKACC International Conference on Control, Loughborough, United Kingdom. pp. 19-24. doi:10.1109/CONTROL . 2014.6915108.

Fonod, R., Henry, D., Charbonnel, C., Bornschlegl, E., 2015. Position and attitude model-based thruster fault diagnosis: A comparison study. Journal of Guidance, Control and Dynamics 38, 1012-1026. doi:10.2514/1.G000309. 
Fu, Y.P., Cheng, Y.H., Jiang, B., Yang, M.K., 2011. Fault tolerant control with on-line control allocation for flexible satellite attitude control system, in: 2nd International Conference on Intelligent Control and Information Processing, IEEE, Piscataway, NJ. pp. 42-46. doi:10. 1109/ICICIP. 2011.6008195.

Gao, Z., Antsaklis, P., 1991. Stability of the pseudo-inverse method for reconfigurable control systems. International Journal of Control 53, 717-729. doi:10.1080/00207179108953643.

Grenaille, S., Henry, D., Zolghadri, A., 2004. Fault diagnosis in satellites using $H_{\infty}$ estimators, in: International Conference on Systems, Man and Cybernetics, IEEE, The Hague, NL. pp. 5195-5200. doi:10.1109/ICSMC. 2004.1401019.

Härkegård, O., 2003. Backstepping and Control Allocation with Applications to Flight Control. Linköping studies in science and technology. thesis no 820. Linköping University. Linköping, Sweden.

Hartley, E.N., Trodden, P.A., Richards, A.G., Maciejowski, J.M., 2012. Model predictive control system design and implementation for spacecraft rendezvous. Control Engineering Practice 20, 695-713. doi:10.1016/j.conengprac.2012.03.009.

HARVD - Final Presentation, 2011. GMV and Thales Alenia Space and Swedish Space Corporation and SENER and jenaoptronik and INTA, Final presentation, December 2011.

Henry, D., 2008a. Fault diagnosis of microscope satellite thrusters using $H_{\infty} / H_{-}$filters. Journal of Guidance, Control, and Dynamics 31, 699-711. doi:10.2514/1.31003.

Henry, D., 2008b. From fault diagnosis to recovery actions for aeronautic and aerospace missions: A model-based point of view, in: 23rd IAR Workshop on Advanced Control and Diagnosis, Coventry, UK. pp. 13-19.

Henry, D., Olive, X., Bornschlegl, E., 2011. A model-based solution for fault diagnosis of thruster faults: Application to the rendezvous phase of the Mars Sample Return mission, in: 4th European Conference for Aerospace Sciences (EUCASS), St. Petersburg, Russian Federation. doi:10.1051/eucass/201306423.

Jiang, J., 1994. Design of reconfigurable control systems using eigenstructure assignments. International Journal of Control 59, 395-410. doi:10.1080/00207179408923083.

Jin, H.P., Wiktor, P., DeBra, D., 1995. An optimal thruster configuration design and evaluation for quick step. Control Engineering Practice 3, 1113-1118. doi:10.1016/0967-0661 (95) 00104-3.

Jin, J., Park, B., Park, Y., Tahk, M.J., 2006. Attitude control of a satellite with redundant thrusters. Aerospace Science and Technology 10, 644-651. doi:10.1016/j . ast.2006.04.005. 
Johansen, T.A., Fossen, T.I., 2013. Control allocation - survey. Automatica 49, 1087-1103. doi:10.1016/j.automatica.2013.01.035.

Josh, S., 1987. Design of failure accommodating multiloop LQG-type controllers. IEEE Transactions on Automatic Control 32, 740-741. doi:10.1109/TAC.1987.1104704.

Konopliv, A.S., Sjogren, W.L., 1995. The JPL Mars gravity field, Mars50c, based upon Viking and Mariner 9 Doppler tracking data. Technical Report. NASA Jet Propulsion Laboratory.

LePeuvédic, C., Charbonnel, C., Henry, D., Strippoli, L., Ankersen, F., 2014. Fault tolerant control design for terminal rendezvous around mars, in: 9th International ESA Conference on GNC, Portugal.

Looze, D., Weiss, J., Eterno, J., Barett, N., 1985. An automatic redesign approach for restructurable control systems. IEEE Control System Magazine 5, 16-22. doi:10.1109/MCS.1985. 1104940.

Maciejowski, J., 2002. Predictive Control with Constraints. Prentice-Hall, Harlow, England.

Noura, H., Theilliol, D., Ponsart, J., Chamseddine, A., 2009. Fault-Tolerant Control Systems: Design and Practical Applications. Springer Verlag, London.

Oppenheimer, M., Doman, D., Bolender, M., 2010. Control allocation, in: Levine, W.S. (Ed.), The control handbook, control system applications (2nd ed., Chapter 8). CRC Press, Boca Raton, FL.

Ostroff, A., 1985. Techniques for accommodating control effector failures on a mildly statically unstable airplane, in: Proceedings of the American Control Conference, pp. 903-906.

Page, A., Steinberg, M., 2002. High-fidelity simulation testing of control allocation methods, in: AIAA Guidance, Navigation and Control Conference and Exhibit, AIAA, Monterey, California. doi:10.2514/6.2002-4547.

Patton, R., Frank, P., Clark, R., 2000. Issues of fault diagnosis for dynamic systems. Springer, London.

Patton, R., Uppal, F., Simani, S., Polle, B., 2006. A monte carlo analysis and design for FDI of a satellite attitude control system, in: Proceedings of SAFEPROCESS'2006, IFAC, Beijing, China. pp. 1393-1398. doi:10.3182/20060829-4-CN-2909.00220.

Patton, R., Uppal, F., Simani, S., Polle, B., 2008. Reliable fault diagnosis scheme for a spacecraft attitude control system. Proceedings of the Institution of Mechanical Engineers Part O: Journal of Risk and Reliability 222, 139-152. doi:10.1243/1748006X JRR98.

Patton, R., Uppal, F., Simani, S., Polle, B., 2010. Robust FDI applied to thruster faults of a satellite system. Control Engineering Practice 18, 1093-1109. doi:10.1016/j .conengprac . 2009.04 .011$. 
Pettazzi, L., Lanzon, A., Theil, S., Finzi, A., 2009. Design of robust drag-free controllers with given structure. Journal of Guidance, Control, and Dynamics 32, 1609-1621. doi:10.2514/ 1.40279 .

Posch, A., Schwientek, A., Sommer, J., Fichter, W., 2013. Model-based on-board realtime thruster fault monitoring, in: Proceedings of IFAC Symposium on Automatic Control in Aerospace, Würzburg, Germany. pp. 553-558. doi:10.3182/20130902-5-DE-2040.00080.

Sidi, M.J., 1997. Spacecraft Dynamics and Control. Cambridge University Press, Cambridge, England, UK.

Staroswiecki, M., 2005. Fault tolerant control: the pseudo-inverse method revisited, in: Proceedings of the 16th IFAC World Congress, IFAC, Prague, Czech Republic. pp. 1871-1871. doi:10.3182/20050703-6-CZ-1902.01872.

Staroswiecki, M., Yang, H., Jiang, B., 2007. Progressive accommodation of parametric faults in linear quadratic control. Automatica 43, 2070-2076. doi:10.1016/j.automatica. 2007. 04.016.

Tafazoli, M., 2009. A study of on-orbit spacecraft failures. Acta Astronautica 64, 195-205. doi:10.1016/j.actaastro.2008.07.019.

Tao, G., Chen, S., Joshi, S., 2002. An adaptive control scheme for systems with unknown actuator failures. Automatica 38, 1027-1034. doi:10.1016/S0005-1098(02)00018-3.

Veillette, R., 1995. Reliable linear-quadratic state-feedback control. Automatica 31, 137-143. doi:10.1016/0005-1098(94)E0045-J.

Wu, Q., Saif, M., 2009. Model-based robust fault diagnosis for satellite control systems using learning and sliding mode approaches. Journal of computers 4, 1022-1032. doi:10.4304/ jсp.4.10.1022-1032.

Yang, H., Jiang, B., Cocquempot, V., 2012. Supervisory fault tolerant control with integrated fault detection and isolation: a switched system approach. International Journal of Applied Mathematics and Computer Science 22, 87-97. doi:10.2478/v10006-012-0006-9.

Zhang, X., Parisini, T., Polycarpou, M., 2004. Adaptive fault-tolerant control of nonlinear uncertain systems: An information based diagnostic approach. IEEE Transactions on Automatic Control 49, 1259-1274. doi:10.1109/TAC.2004.832201.

Zhang, Y., Jiang, J., 2001. Integrated active fault-tolerant control using imm approach. IEEE Transactions on Aerospace and Electronic Systems 37, 1221-1235. doi:10.1109/7.976961.

Zhang, Y., Jiang, J., 2008. Bibliographical review on reconfigurable fault-tolerant control systems. Annual Reviews in Control 32, 229-252. doi:10.1016/j .arcontrol.2008.03.008. 
Zhao, Q., Jiang, J., 1998. Reliable state feedback control systems design against actuator failures. Automatica 34, 1267-1272. doi:10.1016/S0005-1098(98)00072-7.

Zhou, K., Khargonekar, P.P., 1988. Robust stabilization of linear systems with norm-bounded time-varying uncertainty. Systems \& Control Letters 10, 17-20. 